\title{
Stability Criterion for the Centrifugal Instability of Surface Intensified Anticyclones
}

\author{
EUNOK Yim AND ALEXANDRE STEGNER \\ Laboratoire de Météorologie Dynamique, École Polytechnique, Palaiseau, France \\ PAUL BiLlant \\ LadHyX, CNRS, École Polytechnique, Palaiseau, France
}

(Manuscript received 28 May 2018, in final form 24 November 2018)

\begin{abstract}
We investigate the linear stability of intense baroclinic anticyclones, with a particular focus on the centrifugal (inertial) instability. Various vertical and radial velocity profiles are studied. The vertical profiles are such that the velocity is maximum at the surface. These profiles correspond to oceanic eddies such as submesoscale mixed-layer eddies or intense mesoscale eddies in the upper thermocline. The results show that the main characteristics of the centrifugal instability (growth rate, vertical wavelength) depend weakly on the baroclinic structure of the anticyclone. The dominant azimuthal wavenumber is $m=2$ for small Burger number $(\mathrm{Bu})$ and $m=1$ for higher $\mathrm{Bu}$, where $\mathrm{Bu}$ is the square root of the ratio of the deformation radius $R_{d}$ over the characteristic eddy radius $R_{\max }$ where the velocity is maximum. The marginal stability limits of the centrifugal instability for the different velocity profiles collapse approximately on a single curve in the parameter space $(\mathrm{Ro}, \mathrm{Bu})$, where $\mathrm{Ro}=V_{\max } /\left(f R_{\max }\right)$ is the Rossby number, with $V_{\max }$ being the maximum velocity. By means of an asymptotic analysis for short vertical wavelength, an explicit prediction for the marginal stability limit is derived for a wide range of velocity profiles. We then suggest to use, for most of oceanic anticyclones, the instability criterion valid for a Gaussian eddy: $\sqrt{\mathrm{Bu}}=R_{d} / R_{\max } \leq(0.23 / \sqrt{\mathrm{Ek}})(\mathrm{Ro}+0.3)^{2} / \sqrt{|\mathrm{Ro}|}$, where $\mathrm{Ek}=\nu / f H^{2}$ is the Ekman number, $H$ is the eddy depth, and $\nu$ is the turbulent viscosity at the ocean surface. Some baroclinic anticyclones can remain stable even if they have a core region of negative absolute vorticity provided that they are small enough. This formula explains the few observations of intense anticyclonic eddies having a negative core vorticity around $-1.5 f$.
\end{abstract}

\section{Introduction}

A wide variety of intense surface eddies are now ubiquitously observed in the oceans and coastal areas from synthetic-aperture radar (SAR) images (Johannessen et al. 1994; Munk et al. 2000) and coastal high-frequency radar (HFR) current measurements (Chavanne et al. 2010; Paduan and Washburn 2013; Schaeffer et al. 2017). These small-scale surface eddies $(5-20 \mathrm{~km})$ were not accessible before with traditional ocean sampling. They may be generated by a number of mechanisms, including vertical mixing, short-wavelength secondary instabilities, frontal shear instabilities, specific wind forcing events, or coastal boundary layer detachment. Because of their small scales, the relative vorticity of these eddies may reach finite values (Hasegawa et al. 2004; Schaeffer

Corresponding author: Eunok Yim, eunok.yim@ladhyx. polytechnique.fr et al. 2017). Such vortex structures are also observed in numerical models when increasing the spatial resolution of regional or coastal models. For instance, the highresolution Regional Ocean Modeling System (ROMS) simulations of an idealized California Current system (Capet et al. 2008) exhibit a wide variety of submesoscale filaments or eddies with intense vertical vorticity and a large spectra of vertical velocity, in contrast to mesoscale eddies. Other primitive equation simulations of unstable oceanic currents or fronts at high resolution (in both horizontal and vertical directions) exhibit also large Rossby number structures in the surface layer (Klein et al. 2008; Gula et al. 2015). Even mesoscale eddies with a radius of the same order or larger than the local deformation radius could sometime reach intense vorticity values with a negative potential vorticity in the core (Chavanne et al. 2010; Ioannou et al. 2017).

Unlike turbulent microscale flows, these intense eddies are strongly influenced by Earth's rotation. According to 
Kloosterziel and van Heijst (1991) or Mutabazi et al. (1992), an anticyclonic circular and barotropic eddy is unstable to three-dimensional perturbations if the generalized Rayleigh discriminant is negative somewhere in the flow $\phi(r)=\left[\zeta_{z}(r)+f\right](2 V / r+f)<0$, where $V(r)$ is the azimuthal velocity, $f$ is the Coriolis parameter, and $\zeta_{z}(r)=\partial_{r} V+V / r$ is the vertical vorticity. This widely used criterion is a sufficient condition for inviscid threedimensional instabilities as demonstrated by short vertical wavelength WKB analyses (Leblanc and Cambon 1997; Sipp and Jacquin 2000; Billant and Gallaire 2005). To be satisfied, a region of negative absolute vorticity $\zeta+f<0$ (i.e., negative potential vorticity) should exist. However, the direct application of the generalized Rayleigh criterion to oceanic eddies might be problematic since it does not take into account the stratification and the dissipation. Indeed, the stratification induces a low wavenumber cutoff (confining the instability to wavelengths below a threshold) for the inertial instability of jets (Plougonven and Zeitlin 2009) or circular eddies (Billant and Gallaire 2005; Kloosterziel et al. 2007; Lazar et al. 2013a). Short vertical wavelength perturbations are also damped by the vertical dissipation, reducing their growth rate. Therefore, intense oceanic vortices may be closer to the marginal stability limit than expected and the generalized Rayleigh criterion can greatly overestimate the unstable region in the parameter space. One of the main results of Lazar et al. (2013a) was to provide a stronger instability criterion for the inertial instability by taking into account both the stratification and the dissipation. They have shown that the unstable area in the parameter space is reduced in the case of barotropic anticyclonic eddies confined in a thin and strongly stratified fluid layer $(N / f \gg 1$, where $N$ is the Brunt-Väisälä frequency associated with a linear background stratification). Close to the marginal stability limit, the growth rates are controlled by the vertical dissipation and are insensitive to the velocity or the vorticity profile if the intensity of the barotropic eddy is characterized by the vortex Rossby number $\mathrm{Ro}=V_{\max } /\left(f R_{\max }\right)$ instead of the relative core vorticity $\zeta(0) / f$. Besides, Lazar et al. (2013b) have derived an analytical marginal stability limit for the idealized Rankine vortex. This simple stability equation, which depends only on the three dimensionless parameters (the Rossby, Burger, and Ekman numbers), appears to be relevant for a wide variety of velocity profiles and can be used to build a "first guess" stability diagram for the inertial destabilization of intense barotropic anticyclones within a viscous and stratified oceanic layer. Large-scale laboratory experiments on intense vortices in thin stratified layers agree well with this analytical stability threshold (Lazar et al. 2013b). Nevertheless, it is limited because of two hypotheses assumed by Lazar et al. (2013b): the barotropic (i.e., columnar) structure of the basic eddy and the axisymmetry of the unstable modes.

However, both meso- or submesoscale oceanic anticyclones are generally baroclinic; in other words, the azimuthal velocity is not uniform along the vertical, especially in the case of surface intensified eddies. Hence, we should determine how the vertical structure of the eddy affects the growth rate and the wavelength selection of the inertial instability compared to columnar vortices. Besides, the centrifugal instability is most unstable for $m=0$ in the inviscid limit but it can destabilize also nonzero azimuthal wavenumbers as first shown by Smyth and McWilliams (1998) for columnar vortices. Using short vertical wavelength asymptotics, Billant and Gallaire (2005) have derived a generalized Rayleigh criterion valid not only for axisymmetric perturbations but also for asymmetric ones. In the presence of stratification and viscous dissipations, which damp low and high vertical wavenumbers, respectively, the asymmetric centrifugal instabilities can become dominant over the axisymmetric one. In particular, linear stability analyses performed on columnar vortices in stratified-rotating fluid (Billant et al. 2004), on isolated anticyclones in two-layer rotating shallow water model (Lahaye and Zeitlin 2015), or on isolated pancake vortices in a continuously stratified fluid (Yim and Billant 2016; Yim et al. 2016) have shown that the most unstable modes of the inertial instability can be asymmetric close to the marginal stability limit. Hence, the main goal of the present study is to obtain, as for the axisymmetric inertial modes on barotropic eddies (Lazar et al. 2013a), a simple marginal stability criterion for the asymmetric inertial instability of baroclinic surface anticyclones.

To this end, we address in this paper the linear stability of a surface axisymmetric anticyclone in a rotating and linearly stratified fluid using the Navier-Stokes equation with a standard Laplacian dissipation. We introduce a solid free-slip wall condition at the surface where the vortex intensity is maximum in order to mimic the flat ocean surface. This specific boundary condition is relevant only for surface eddies, and we exclude from the scope of our investigation intrathermocline eddies or meddies as considered by Nguyen et al. (2012), Hua et al. (2013), Yim et al. (2016), Facchini and Le Bars (2016), Sutyrin and Radko (2017), Reinaud (2017), and Mahdinia et al. (2017). Unlike these previous studies dedicated only to Gaussian lenses, we will study various radial and vertical profiles in order to extract some general stability properties which are not profile dependent. Only linear stability results on the centrifugal instability will be presented although other types of 
instability can occur. Because of the baroclinic structure of the eddy, the barotropic $m=2$ shear instability will exist only for submesoscale structures. Yim et al. (2016) showed that Gaussian pancake vortices having small Rossby number are barotropically unstable when the characteristic radius is below $1 / 7$ of the deformation radius $R_{d}$. On the other hand, baroclinic instability will affect only large-scale eddies. For instance, Yim et al. (2016) and Mahdinia et al. (2017) showed that geostrophic Gaussian lenses are unstable to mixed barotropic/baroclinic instability when the radius exceeds $1.3 R_{d}$. For $m=1$, there also exists an instability called Gent-McWilliams instability that bends the vortex. However, for finite Rossby number sufficiently above the centrifugal instability threshold, these other instabilities are subdominant.

The paper is organized as follows. We first describe in section 2 the general class of 3D circular surface eddies considered as base state. The methods used to solve the generalized eigenvalue problem for the full NavierStokes equations are given in section 3. After introducing the asymptotic results for centrifugal instability in section 4 , the results of the stability analysis are presented in section 5. We first investigate in section 5a the impact of the vertical eddy structure (baroclinic/ barotropic) on the inertial instability of intense anticyclones. Besides, a quantitative comparisons between the full 3D stability analysis and the asymptotic solutions obtained for columnar eddies are provided. Then, we investigate in section $5 b$ the impact of the radial velocity profiles on the inertial instability and build the stability diagrams in the parameter space of the Burger number and the vortex Rossby number for distinct Ekman numbers. By means of an asymptotic analysis, an analytical stability criterion is derived that is in good agreement with the numerical stability analysis. Finally we summarize our results and discuss their applications to oceanic eddies in section 6 .

\section{Structure of the surface intensified anticyclones}

\section{a. Velocity and vorticity profiles}

The angular velocity $\Omega(r, z)$ of the isolated circular vortices considered herein is prescribed by two dimensionless functions $F_{\alpha}(\hat{r})$ and $G(\hat{z})$ :

$$
\frac{V_{\theta}}{r}=\Omega(r, z)=\Omega_{0} F_{\alpha}(\hat{r}) G(\hat{z}),
$$

with $\hat{r}=r / R_{\max }$ being the dimensionless radius and $\hat{z}=z / H$ the dimensionless depth of the vortex. The typical vortex depth is $H$ and the characteristic vortex radius $R_{\max }$ corresponds to the radius where the azimuthal velocity $V_{\theta}$ is maximum $V_{\theta}\left(R_{\max }\right)=V_{\max }$. We use for the radial distribution of the angular velocity a general class of profiles parameterized by the steepness parameter $\alpha$ as

$$
F_{\alpha}(\hat{r})=\exp \left(-\frac{1}{\alpha} \hat{r}^{\alpha}\right) .
$$

This equation describes a wide range of profiles having smooth $(1<\alpha \leq 2)$ or steep velocity gradients (large $\alpha$ ) with always a vanishing circulation for large radii. The case $\alpha=2$ corresponds to a Gaussian velocity profile. The radial $\zeta_{r}$ and vertical $\zeta_{z}$ vorticity components of such circular vortices are given by

$$
\begin{aligned}
& \zeta_{r}=-\partial_{z} V_{\theta}=-\frac{r}{H} \Omega(r, z) \frac{G^{\prime}(\hat{z})}{G(\hat{z})}, \\
& \zeta_{z}=\frac{1}{r} \frac{\partial r^{2} \Omega}{\partial r}=\Omega\left(2-\hat{r}^{\alpha}\right) .
\end{aligned}
$$

The relative vorticity $\zeta / f$ on the axis $r=0$ is directed along the vertical axis and given by

$$
\frac{\zeta_{z}(0)}{f}=\frac{2 \Omega_{0}}{f}=2 e^{1 / \alpha} \operatorname{Ro},
$$

where

$$
\mathrm{Ro}=\frac{V_{\max }}{f R_{\max }}=\frac{\Omega_{0}}{f} e^{-1 / \alpha}
$$

is the vortex Rossby number. Anticyclonic (cyclonic) eddies correspond to negative (positive) values of Ro. The inviscid stability of such class of vortices when $G(\hat{z})=1$ has been studied by Carton and McWilliams (1989), Carnevale and Kloosterziel (1994), and Orlandi and Carnevale (1999) for purely two dimensional flows, by Stegner and Dritschel (2000) for one-layer quasigeostrophic (QG) and shallow-water flows, and by Smyth and McWilliams (1998) for 3D stratified-rotating flows. In the $2 \mathrm{D}$ limit, the vortex is unstable to the shear (i.e., barotropic) instability when the steepness parameter is larger than $\alpha_{c}=1.85$. For $\alpha=2$, only the azimuthal wavenumber $m=2$ is unstable. In the shallowwater one-layer QG framework, the barotropic instability tends to weaken when the ratio of the deformation radius to the vortex radius decreases (Stegner and Dritschel 2000). In contrast, for two-layer quasigeostrophic vortices, the baroclinic instability is enhanced when the vortex size increases (Ikeda 1981; Flierl 1988; Helfrich and Send 1988; Benilov 2003). In 3D stratified rotating flows, the centrifugal instability can also occur (Smyth and McWilliams 1998). In nonrotating flows, Billant and Gallaire (2005) have shown that the azimuthal wavenumbers in the range $|m|<2 \sqrt{\alpha}$ are centrifugally unstable but the mode $m=0$ is the most unstable in the inviscid 

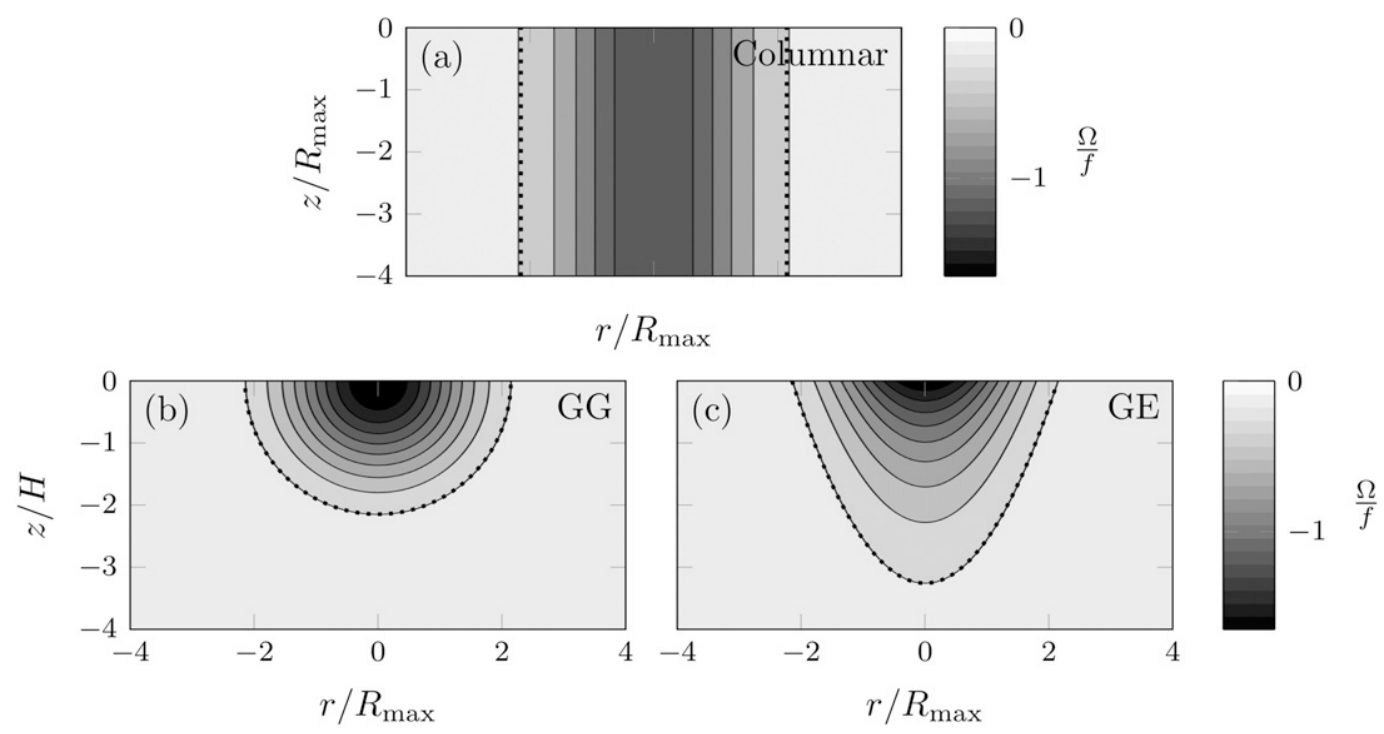

FIG. 1. Vertical cross sections of the relative angular velocity of (a) Gaussian columnar, (b) GG, and (c) GE anticyclones when Ro $=-1$. Dotted lines indicate the limit of the base vortex where $\Omega=0.1 \Omega_{0}$.

limit. In addition, the azimuthal wavenumber $m=1$ can be unstable to the Gent and McWilliams instability when the fluid is stratified-rotating (Gent and McWilliams 1986; Yim and Billant 2015).

Along the vertical, we use different profiles $G(\hat{z})$ corresponding to a barotropic columnar vortex $G_{C}(\hat{z})=1$ (reference case), a Gaussian profile $G_{G}(\hat{z})=e^{-\hat{z}^{2} / 2}$, and an exponential profile $G_{E}(\hat{z})=e^{\hat{z} / \sqrt{2}}$ for surface lenses. Vertical cross sections of the angular velocity of the Gaussian-columnar vortex $\left[\Omega(r, z)=\Omega_{0} F_{2}(\hat{r}) G_{C}(\hat{z})\right]$, the Gaussian-Gaussian (GG) vortex $\left[\Omega(r, z)=\Omega_{0} F_{2}(\hat{r}) G_{G}(\hat{z})\right]$, and the Gaussian-exponential (GE) vortex $[\Omega(r, z)=$ $\left.\Omega_{0} F_{2}(\hat{r}) G_{E}(\hat{z})\right]$ are plotted in Fig. 1 . The dotted lines represents the contour $\Omega=0.1 \Omega_{0}$, indicating the eddy extent. The axial and radial vorticity components for the GG and GE vortices are shown in Fig. 2. Note that the exponential profile GE has a nonzero shear at the surface. The resulting Ekman pumping can be estimated as $w_{E} \sim V_{\max } \delta_{E} / H$, where $\delta_{E}=\sqrt{2 \nu / f}$ is the Ekman layer depth with $\nu$ being the viscosity. For the typical parameters investigated in the following, the associated decay rate scaled by $|f|$ is $w_{E} /(H|f|) \sim O\left(10^{-2}\right)$. It is expected that the instabilities will not be influenced by this effect as long as their scaled growth rates $\omega_{i} /|f|$ are larger compared to this typical decay rate.

\section{b. Associated density anomaly}

In addition, the angular velocity (1) should satisfy the thermal-wind balance:

$$
\frac{\partial}{\partial z}\left(r \Omega^{2}+f r \Omega\right)=-\frac{g}{\rho_{0}} \frac{\partial}{\partial r} \rho_{a}
$$

where $\rho_{a}$ is the density anomaly due to the eddy velocity. Therefore, the total density is given by $\rho_{t}=\rho_{0}+\bar{\rho} z+\rho_{a}$, where $\rho_{0}$ is the reference density and $\bar{\rho}=-N_{0}^{2} \rho_{0} / g$ is the stable background density gradient, with $N_{0}$ being the constant Brunt-Väisälä frequency of the unperturbed flow (i.e., with no eddy).

For a barotropic columnar vortex, the isopycnals are always flat since there is no vertical variation of the velocity field, while for the baroclinic GG eddy the base density anomaly is given by

$$
\rho_{a}(r, z)=-\frac{\rho_{0}}{g} z\left(\frac{R_{\max }}{H}\right)^{2} \Omega(\Omega+f) .
$$

To make this density anomaly dimensionless, we compare it to the vertical variation of the background density flow along the eddy depth $H(\Delta \rho=\bar{\rho} H)$ :

$$
\begin{aligned}
\frac{\rho_{a}(r, z)}{\Delta \rho} & =-\frac{z}{H} \frac{1}{N_{0}^{2}}\left(\frac{R_{\max }}{H}\right)^{2} \Omega(\Omega+f) \\
& =-\frac{\operatorname{Ro}}{\mathrm{Bu}} \sqrt{e} \hat{z}\left[\operatorname{Ro} \sqrt{e} F_{2}(\hat{r}) G_{G}(\hat{z})+1\right] F_{2}(\hat{r}) G_{G}(\hat{z})
\end{aligned}
$$

where

$$
\mathrm{Bu}=\frac{N_{0}^{2} H^{2}}{f^{2} R_{\max }^{2}}=\left(\frac{R_{d}}{R_{\max }}\right)^{2}
$$

is the Burger number and $R_{d}=N_{0} H / f$ is the baroclinic deformation radius. Two examples of dimensionless density anomalies are shown in Fig. 3 for intense 

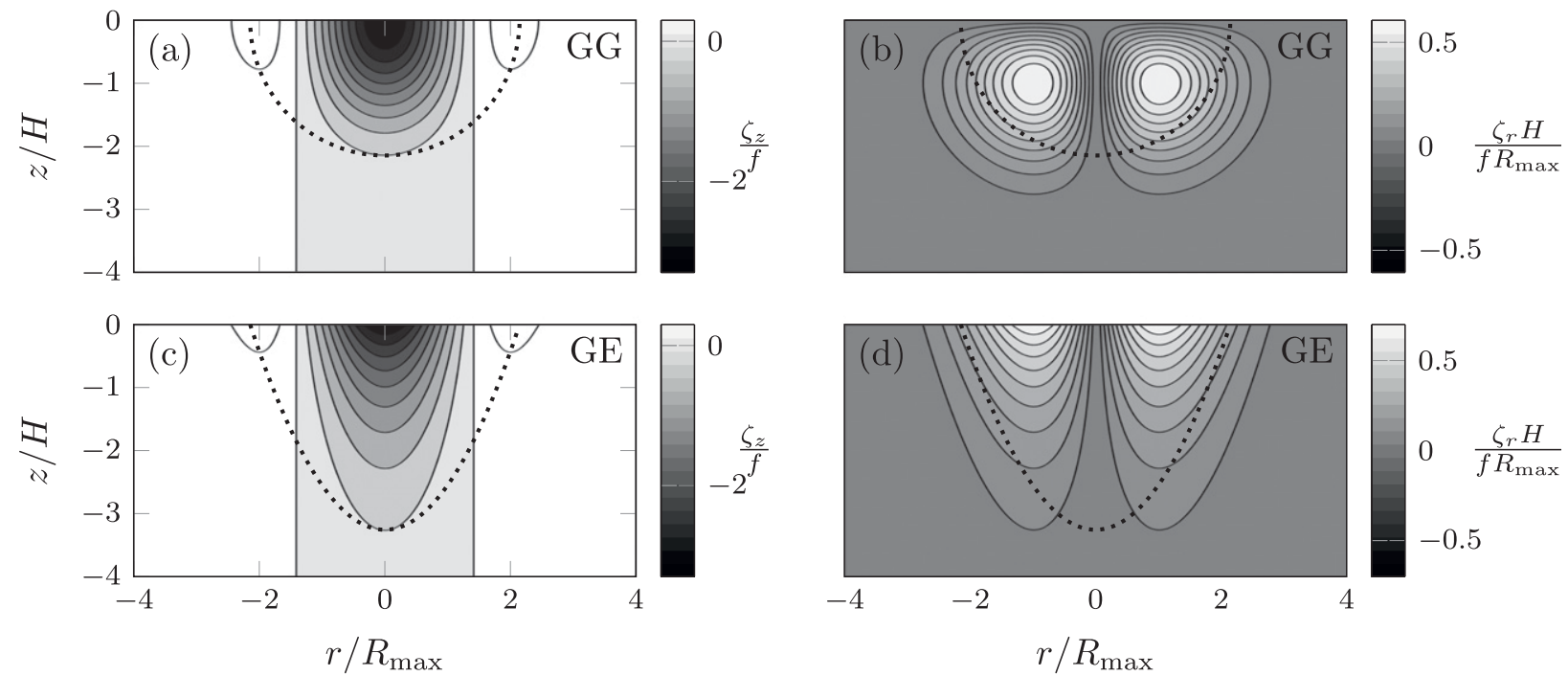

FIG. 2. Vertical cross sections of the relative (left) axial and (right) radial vorticity components for the (a),(b) GG and (c),(d) GE anticyclones for Ro $=-1$. Dotted lines indicate the limit of the base vortex where $\Omega=0.1 \Omega_{0}$.

submesoscale $(\mathrm{Ro}=-1.5, \mathrm{Bu}=4$; Fig. 3a) and strong mesoscale $(\mathrm{Ro}=-0.5, \mathrm{Bu}=1$; Fig. $3 \mathrm{~b}) \mathrm{GG}$ anticyclones.

For a baroclinic GE vortex, the base density anomaly is given by

$$
\rho_{a}(r, z)=\frac{\rho_{0}}{g} \frac{R_{\max }^{2}}{H \sqrt{2}} \Omega(\Omega+f)
$$

After nondimensionalization, this can be rewritten as

$$
\frac{\rho_{a}(r, z)}{\Delta \rho}=\frac{\mathrm{Ro}}{\mathrm{Bu}} \sqrt{e / 2}\left[\operatorname{Ro} \sqrt{e} F_{2}(\hat{r}) G_{E}(\hat{z})+1\right] F_{2}(\hat{r}) G_{E}(\hat{z}) .
$$

Unlike the GG eddy, the density anomaly for the GE vortex is maximum at the surface $(z=0)$ :
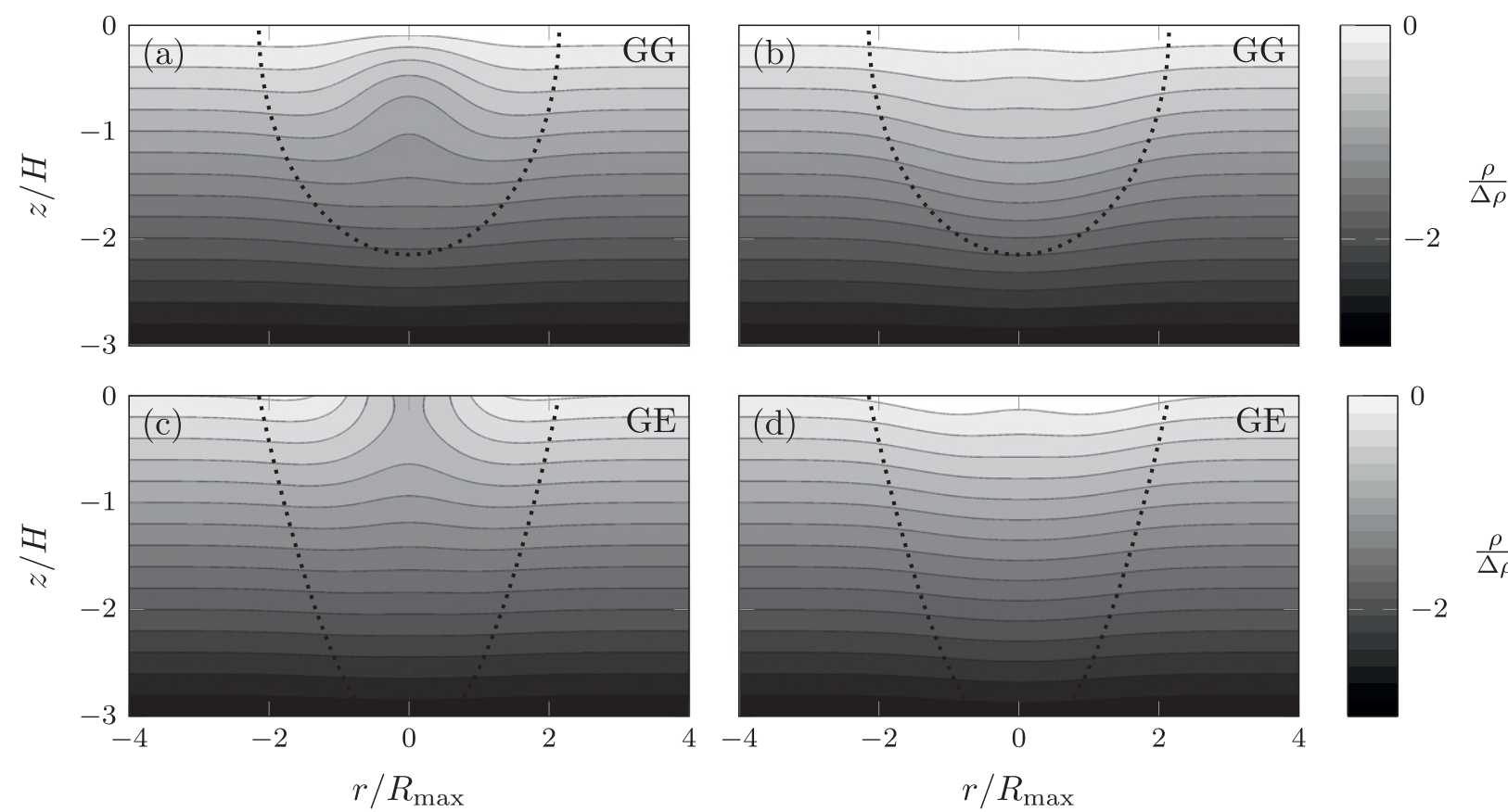

FIG. 3. Vertical cross sections of the total density fields $\rho_{t} / \Delta \rho$ of the (top) GG and (bottom) GE anticyclones when (a), (c) Ro $=-1.5$ and $\mathrm{Bu}=4$ and (b),(d) Ro $=-0.5$ and $\mathrm{Bu}=1$. Dotted lines indicate the limit of the base vortex where $\Omega=0.1 \Omega_{0}$. 

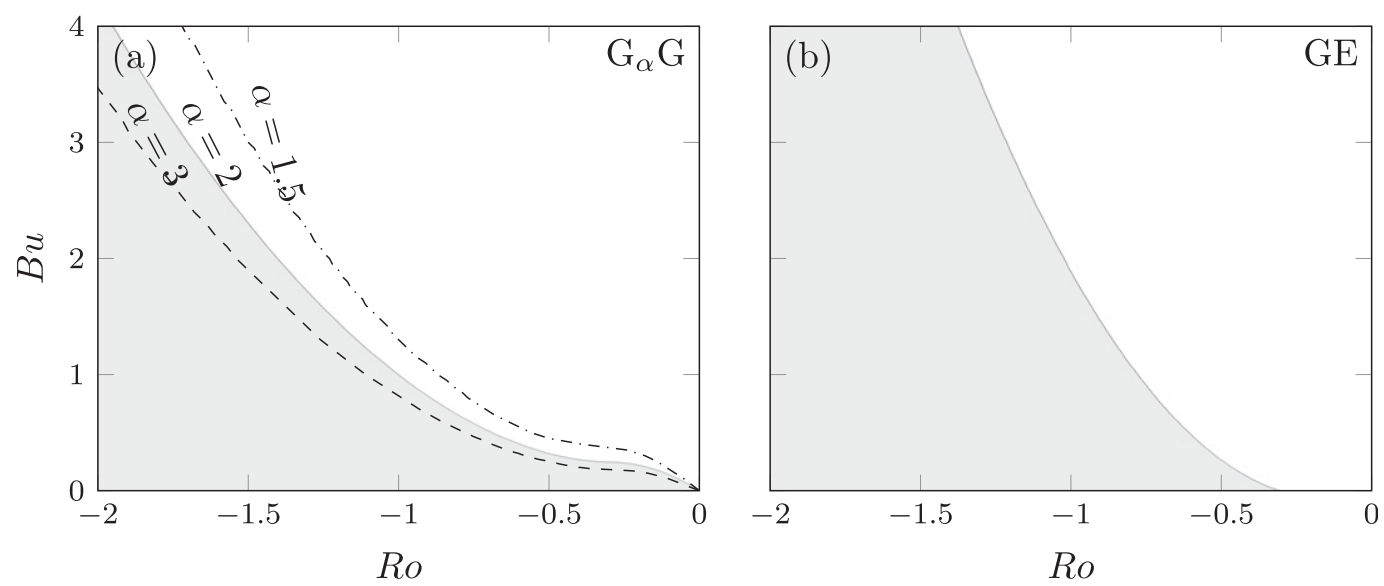

FIG. 4. Domain of the gravitational instability in the parameter space (Ro, Bu) for (a) $\mathrm{G}_{\alpha} \mathrm{G}$ and (b) GE anticyclones. The unstable region is shaded. In (a), the limits for various steepness parameters are plotted: $\alpha=1.5$ (dash-dotted line), $\alpha=2$ (solid line), and $\alpha=3$ (dashed line). In (b), $\alpha$ is fixed to $\alpha=2$.

$$
\frac{\rho_{a}(r, 0)}{\Delta \rho}=\frac{\mathrm{Ro}}{\mathrm{Bu}} \sqrt{e / 2}\left(\sqrt{e} \operatorname{Ro} e^{-\hat{r}^{2} / 2}+1\right) e^{-\hat{r}^{2} / 2}
$$

Because of the cyclogeostrophic balance, the sign of the surface density anomaly depends on the vortex intensity. For low Rossby numbers $\left(-e^{-1 / 2}<\right.$ Ro $\left.<0\right)$, the density anomaly is negative while for intense anticyclones (Ro $\lesssim-0.6)$ the density anomaly is positive at $r=0$. In all the cases, the isopycnals outcrop at the free surface as seen in Figs. 3c and 3d.

For the values of $\alpha$ different from 2, the density anomaly cannot be derived analytically and has to be computed numerically.

Depending on $\mathrm{Ro}$ and $\mathrm{Bu}$, the density anomaly can be strong and eventually make the GG and GE eddies statically unstable (gravitational instability) (Negretti and Billant 2013). This occurs when the vertical gradient of the total density becomes positive somewhere in the vortex: $\partial \rho_{t} / \partial z>0$. Figure 4 shows the domains of this instability in the parameter space $(\mathrm{Ro}, \mathrm{Bu})$ for the two profiles GG and GE for different values of the steepness parameter $\alpha$. The unstable domain for the $\mathrm{GE}$ vortex tends to be wider than for the $\mathrm{G}_{\alpha} \mathrm{G}$ vortex. However, the domain of instability depends on $\alpha$ for the $\mathrm{G}_{\alpha} \mathrm{G}$ (Fig. 4a). In the following, we will only consider eddies that are stable to the gravitational instability.

Because of the vertical structure of these eddies and their background stratification, the baroclinic instability could also induce destabilization of the $\mathrm{G}_{\alpha} \mathrm{G}$ or the GE eddies. The linear stability of 3D lenses was studied by Nguyen et al. (2012) with the QG stratified model, and by Yim et al. (2016) and Mahdinia et al. (2017) within the Boussinesq framework. We could transpose these stability analyses performed for these 3D Gaussian lenses to surface intensified eddies by considering only the vertically symmetric modes. For large eddies, when the eddy radius $R_{\max }$ slightly exceeds the baroclinic deformation radius $R_{d}$, and small Rossby number, the $m=2$ baroclinic mode is the dominant unstable mode both in QG and the full 3D Boussinesq model. This $m=2$ mode is in fact a mixed mode between the baroclinic instability and the shear instability as shown by Yim et al. (2016) (see their Fig. 39f). Purely baroclinic modes with higher azimuthal wavenumbers $(m>2)$ may be dominant (Nguyen et al. 2012; Yim et al. 2016) but only for very large eddies (when $\mathrm{Bu}<0.1$ ), which are out of the scope of this study. However, for ageostrophic eddies with finite Rossby numbers, a cyclone-anticyclone asymmetry occurs, and the baroclinic growth rates are slower for anticyclonic Gaussian lenses than their cyclonic counterparts. Yim et al. (2016) give an empirical marginal stability limit for the mixed barotropic-baroclinic mode $(m=2)$ of Gaussian eddies $(\alpha=2)$ having a weak dissipation $(\operatorname{Re}=10000)$ : it is unstable only if the Burger number is below a critical value: $\mathrm{Bu}<\mathrm{Bu}_{c}=1.3 /\left(2.26-\zeta_{0} / f\right)=1.3 /(2.26-$ $\left.2 \mathrm{Ro} e^{1 / 2}\right)$. Hence, mesoscale or submesoscale Gaussian anticyclones (i.e., $\mathrm{Bu} \geq 1$ ) remain baroclinically stable and the centrifugal or symmetric instability can then be the only cause of destabilization for finite Rossby numbers. The full stability analysis performed by Yim et al. (2016) confirms that when it appears, the centrifugal instability dominates the baroclinic $(m=2)$ and the Gent-McWilliams $(m=1)$ instabilities. We have also checked that, for the intense surface intensified (but not too large) anticyclones we studied, the centrifugal instability is indeed the dominant one (see appendix B). 


\section{Formulation of the linear stability analysis problem}

\section{a. Linear eigenvalue problem}

To perform the linear stability analysis of circular surface intensified eddies, we introduce infinitesimal perturbations of velocity $\mathbf{u}^{\prime}=\left(u_{r}^{\prime}, u_{\theta}^{\prime}, u_{z}^{\prime}\right)$, pressure $p^{\prime}$, and density $\rho^{\prime}$ written as

$$
\begin{aligned}
\left(u_{r}^{\prime}, u_{\theta}^{\prime}, u_{z}^{\prime}, p^{\prime}, \rho^{\prime}\right)= & {\left[u_{r}(r, z), u_{\theta}(r, z), u_{z}(r, z), p(r, z),\right.} \\
& \rho(r, z)] e^{-i \omega t+i m \theta}+\text { c.c. },
\end{aligned}
$$

where $m$ is the azimuthal wavenumber and $\omega=\omega_{r}+i \omega_{i}$, with $\omega_{r}$ being the frequency and $\omega_{i}$ the growth rate. We then linearize the Navier-Stokes equations under the Boussinesq approximation around the steady circular solution (1) and the basic density $\rho_{a}$ :

$$
\begin{aligned}
& -i s u_{r}-(2 \Omega+f) u_{\theta} \\
& =-\frac{1}{\rho_{0}} \frac{\partial p}{\partial r}+\nu\left(\nabla^{2} u_{r}-\frac{1}{r^{2}} u_{r}-\frac{2}{r^{2}} i m u_{\theta}\right), \\
& -i s u_{\theta}+\left(\zeta_{z}+f\right) u_{r}+\frac{\partial r \Omega}{\partial z} u_{z} \\
& =-\frac{1}{\rho_{0}} \frac{i m}{r} p+\nu\left(\nabla^{2} u_{\theta}-\frac{1}{r^{2}} u_{\theta}+\frac{2}{r^{2}} i m u_{r}\right),
\end{aligned}
$$

$-i s u_{z}=-\frac{1}{\rho_{0}} \frac{\partial p}{\partial z}-\frac{g}{\rho_{0}} \rho+\nu \nabla^{2} u_{z}$,

$-i s \rho+\frac{\partial \rho_{a}}{\partial r} u_{r}+\frac{\partial \rho_{a}}{\partial z} u_{z}=\frac{\rho_{0}}{g} N_{0}^{2} u_{z}+\kappa \nabla^{2} \rho, \quad$ and

$\frac{1}{r} \frac{\partial r u_{r}}{\partial r}+\frac{1}{r} i m u_{\theta}+\frac{\partial u_{z}}{\partial z}=0$,

where $s=\omega-m \Omega, \nu$ is the viscosity, and $\kappa$ is the diffusivity. This linear eigenvalue problem is governed by five nondimensional numbers: the Burger number $(\mathrm{Bu})$ and the Rossby number (Ro) defined previously in (10) and (6) and the aspect ratio $\delta$, the Ekman number Ek, and the Schmidt number (Sc), defined as follows:

$$
\delta=\frac{H}{R_{\max }}, \quad \mathrm{Ek}=\frac{\nu}{f H^{2}}, \quad \mathrm{Sc}=\frac{\nu}{\kappa} .
$$

In the oceans, the flow is expected to be highly turbulent. Therefore, the Schmidt number Sc is fixed to unity since the turbulent advection at small scales dominates the molecular viscosity and diffusivity. The variables in (15)-(19) are nondimensionalized as follows:

$$
\begin{aligned}
& u_{r}=V_{\max } \hat{u}_{r}, \quad u_{\theta}=V_{\max } \hat{u}_{\theta}, \quad u_{z}=V_{\max } \delta \hat{u}_{z}, \quad r=\hat{r} R_{\max }, \quad z=\hat{z} H, \quad \omega=f \hat{\omega}, \\
& \Omega=V_{\max } R_{\max }^{-1} \hat{\Omega}, \quad p=V_{\max } R_{\max } f \rho_{0} \hat{p}, \quad \rho=V_{\max } f \delta^{-1} \rho_{0} g^{-1} \hat{\rho}, \quad \rho_{a}=V_{\max } f \delta^{-1} \rho_{0} g^{-1} \hat{\rho}_{a},
\end{aligned}
$$

where a hat indicates nondimensional values. This leads to the following:

$$
\begin{gathered}
-i \hat{s} \hat{u}_{r}-(2 \operatorname{Ro} \hat{\Omega}+1) \hat{u}_{\theta} \\
=-\frac{\partial \hat{p}}{\partial \hat{r}}+\operatorname{Ek}\left(\hat{\nabla}^{2} \hat{u}_{r}-\frac{\delta^{2}}{\hat{r}^{2}} \hat{u}_{r}-\frac{2 \delta^{2}}{\hat{r}^{2}} i m \hat{u}_{\theta}\right), \\
-i \hat{u}_{\theta}+(\operatorname{Ro} \hat{\zeta}+1) \hat{u}_{r}+\operatorname{Ro} \frac{\partial \hat{r} \hat{\Omega}}{\partial \hat{z}} \hat{u}_{z} \\
=-\frac{i m}{\hat{r}} \hat{p}+\operatorname{Ek}\left(\hat{\nabla}^{2} \hat{u}_{\theta}-\frac{\delta^{2}}{\hat{r}^{2}} \hat{u}_{\theta}+\frac{\delta^{2}}{\hat{r}^{2}} i m \hat{u}_{r}\right), \\
-i \delta^{2} \hat{s}_{z}=-\frac{\partial \hat{p}}{\partial \hat{z}}-\hat{\rho}+\operatorname{Ek} \delta^{2} \hat{\nabla}^{2} \hat{u}_{z}, \\
-i \hat{s} \hat{\rho}+\operatorname{Ro} \frac{\partial \hat{\rho}_{b}}{\partial \hat{r}} \hat{u}_{r}+\frac{\operatorname{Ro}}{\delta} \frac{\partial \hat{\rho}_{b}}{\partial \hat{z}} \hat{u}_{z}=\frac{\mathrm{Bu}}{\delta} \hat{u}_{z}+\frac{\mathrm{Ek}}{\mathrm{Sc}} \hat{\nabla}^{2} \hat{\rho},
\end{gathered}
$$

$$
\frac{\partial \hat{u}_{r}}{\partial \hat{r}}+\frac{\hat{u}_{r}}{\hat{r}}+\frac{1}{\hat{r}} i m \hat{u}_{\theta}+\frac{\partial \hat{u}_{z}}{\partial \hat{z}}=0,
$$

where $\hat{s}=\hat{\omega}-m \operatorname{Ro} \hat{\Omega}$ and $\hat{\nabla}^{2}=\delta^{2} / \hat{r} \partial / \partial \hat{r}(\hat{r} \partial / \partial \hat{r})-\delta^{2} / \hat{r}^{2} m^{2}$ $+\partial^{2} / \partial \hat{z}^{2}$.

\section{b. Numerical methods}

The numerical method to solve (22)-(26) is similar to the one used by Yim and Billant (2016) and Yim (2015) so that only a brief summary is given here. Equations (22)-(26) are discretized with finite element method using FreeFem ++ (Hecht 2012; Garnaud 2012; Garnaud et al. 2013). Velocity, density, and pressure $\hat{\mathbf{v}}=\left(\hat{u}_{r}\right.$, $\left.\hat{u}_{\theta}, \hat{u}_{z}, \hat{\rho}, \hat{p}\right)$ are approximated with triangular TaylorHood elements (P2, P2, P2, P2, P1), respectively (Elman et al. 2005; Hecht 2012). Unlike Yim and Billant (2016) and Yim et al. (2016), we use P2 elements for the density since the base density cannot be expressed analytically when $\alpha$ is arbitrary. The thermal-wind 
equation (7) is therefore integrated numerically using the same element as for the velocity. The domain is restricted to positive radius $r=\left[0, L_{\max }\right]$ and negative vertical coordinate $z=\left[-Z_{\max }, 0\right]$. A solid wall free-slip boundary condition is applied at $z=0$ : $u_{z}=\rho=0$. At $r=0$ the boundary condition depends on the azimuthal wavenumber $m$ (Batchelor and Gill 1962; Ash and Khorrami 1995; Yim and Billant 2016; Yim et al. 2016). At the boundaries $r=L_{\max }$ and $z=-Z_{\max }$, all the perturbations are set to zero. The resulting discretized equations are written in the form

$$
-i \omega \mathbf{B v}=\mathbf{L v} .
$$

The typical size of the matrices $\mathbf{B}$ and $\mathbf{L}$ is about $\left(3 \times 10^{5}\right) \times\left(3 \times 10^{5}\right)$. The generalized eigenvalue problem (27) is solved with an iterative Krylov-Schur method using the libraries SLEPc and PETSc (Hernandez et al. 2005; Garnaud 2012; Garnaud et al. 2013; Balay et al. 2014). The shift-invert spectral transformation is used to find the most unstable eigenvalues/vectors around shift values. Spurious modes are eliminated by excluding eigenvalues varying by more than $10^{-6}$ between two successive shift values.

\section{Asymptotic results for the centrifugal instability}

\section{a. Inviscid stability criterion}

The standard stability criterion for the centrifugal instability, first established by Rayleigh (1917), for inviscid, homogeneous, and circular flows, has been extended by Solberg (1936) to baroclinic and stratified flows by means of Lagrangian displacement arguments. A fluid parcel is virtually displaced and the resulting momentum balance is computed by invoking angular momentum and density conservations, while the ambient pressure field is assumed to be unchanged. If the fluid parcel further accelerates away from its original position, the flow is expected to be unstable. This generalized Rayleigh criterion for baroclinic circular flows states that the flow is unstable if the total circulation decreases as the radius increases along isopycnals:

$$
\Phi=\left(\zeta_{z}+f\right)(2 \Omega+f)+\zeta_{r}(2 \Omega+f) \frac{\frac{\partial \rho_{t}}{\partial r}}{\frac{\partial \rho_{t}}{\partial z}}<0
$$

somewhere in the flow. Note that (28) has been written in dimensional form and the nondimensionalization [(21)] will be only used later. The same condition has been derived by Eliassen and Kleinschmidt (1957) by using energy arguments. We can remark that (28) when $2 \Omega+f>0$ gives instability when the potential vorticity is negative, a well-known criterion for symmetric instability. If we consider a statically stable flow $\left(N^{2}=-g / \rho_{0} \partial \rho_{t} / \partial z>0\right)$, the above instability criterion is equivalent to

$$
\chi(r, z) N^{2}<r^{2}(2 \Omega+f)^{2}\left(\frac{\partial \Omega}{\partial z}\right)^{2},
$$

where $\chi=\left(\zeta_{z}+f\right)(2 \Omega+f)$ is the standard Rayleigh discriminant for a barotropic circular vortex. In addition to $r$ and $z, \chi$ depends on the Rossby number while the generalized Rayleigh discriminant $\Phi$ or the dimensionless form of (29) depends, a priori, on two dimensionless parameters Ro and $\mathrm{Bu}$. However, if the vertical profile $G(z)$ is Gaussian, the eddy intensity is maximum at the surface so that $\partial \Omega / \partial z=0$. Hence, the inviscid instability criterion in (29) becomes identical to the classical criterion $\min _{r, z}(\chi)<0$ for barotropic eddies (Kloosterziel and van Heijst 1991; Mutabazi et al. 1992). If the vertical profile $G(z)$ is exponential, the marginal stability curve $\min [\Phi(\mathrm{Ro}, \mathrm{Bu})]=0$ deviates from the limit $\min [\chi(\mathrm{Ro})]_{r, z}^{r, z}=0$ for positive Rossby number and small Burger number. However, for negative Rossby number, the marginal stability limit continues to be reached when the absolute vorticity $f+\zeta_{z}$ vanishes at $r=0$, that is, when the Rossby number reaches the critical value $\mathrm{Ro}_{c \infty}=-1 / 2 e^{-1 / \alpha}$, because the baroclinic term in (28) or (29) also vanishes at $r=0$. Therefore, for both GG and GE vortices, the criterion in (28) is equivalent to $\chi<0$ for anticyclones. This means that the centrifugal stability of baroclinic anticyclonic eddies can depend on the stratification only in presence of dissipation.

\section{b. Asymptotic analysis}

The combined effect of dissipation and stratification can be evidenced by using the asymptotic results derived by Billant and Gallaire (2005) and Yim and Billant (2016) for a columnar vortex for large vertical wavenumber $k \gg 1$ and small viscosity $\nu \ll 1$ such that $\nu k^{2}$ is finite. At leading order in $k$, the complex eigenfrequency can be written in dimensional form:

$$
\omega=\omega^{(0)}-\frac{\omega^{(1)} N}{k}-i \nu k^{2}
$$

where

$$
\omega^{(0)}=m \Omega\left(r_{0}\right)+i \sqrt{-\chi\left(r_{0}\right)},
$$




$$
\omega^{(1)}=\frac{(2 n+1) i}{2 \sqrt{2}} \sqrt{\chi^{\prime \prime}\left(r_{0}\right)-2 m^{2} \Omega^{\prime}\left(r_{0}\right)^{2}+2 i m \sqrt{-\chi\left(r_{0}\right)} \Omega^{\prime \prime}\left(r_{0}\right)} \sqrt{\frac{1}{N^{2}}-\frac{1}{\chi\left(r_{0}\right)}},
$$

where $n$ is a nonnegative integer and $r_{0}$ is given by

$$
\chi^{\prime}\left(r_{0}\right)=-2 i m \Omega^{\prime}\left(r_{0}\right) \sqrt{-\chi\left(r_{0}\right)} .
$$

Equation (30) can be rewritten in terms of the Burger and Ekman numbers (Bu and Ek):

$$
\omega=\omega^{(0)}-\frac{\omega^{(1)} \sqrt{\mathrm{Bu}}|f|}{k \delta}-i \mathrm{Ek}|f| H^{2} k^{2},
$$

where the aspect ratio $\delta$ is defined in (20). The most amplified wavenumber $k_{\max }$ is found by solving $\partial \omega_{i} / \partial k=0$ :

$$
k_{\max } H=\left(\frac{\omega_{i}^{(1)} \sqrt{\mathrm{Bu}} R_{\max }}{2 \mathrm{Ek}}\right)^{1 / 3},
$$

where $\omega_{i}^{(j)}$ is the imaginary part of the complex pulsation $\omega^{(j)}$. Substituting (35) in (34) gives the maximum growth rate:

$$
\frac{\omega_{i \max }}{|f|}=\frac{\omega_{i}^{(0)}}{|f|}-3(\mathrm{BuEk})^{1 / 3}\left(\frac{\omega_{i}^{(1)} R_{\max }}{2}\right)^{2 / 3} .
$$

The aspect ratio $\delta$ has disappeared in (36) as expected, for columnar vortices. We have observed a similar behavior for baroclinic eddies for small aspect ratio. According to appendix A, the aspect ratio has indeed almost no effect on the maximum growth rate and most amplified wavenumber for given $\mathrm{Bu}, \mathrm{Ro}$, and $\mathrm{Ek}$ as long as $\delta$ does not exceed unity, which is obviously the case for oceanic eddies. Moreover, we can obtain from (36) an equation for the marginal stability limit $\left(\omega_{i \max }=0\right)$ :

$$
\sqrt{\mathrm{Bu}}=\frac{1}{\sqrt{\mathrm{Ek}}}\left(\frac{\omega_{i}^{(0)}}{3|f|}\right)^{3 / 2}\left(\frac{2}{\omega_{i}^{(1)} R_{\max }}\right) .
$$

In the case of strong stratification $N^{2} \gg \Omega_{0}^{2}$, we can neglect the inverse of the Brunt-Väisälä frequency in (32). Then, the ratio $\mathscr{F}=2\left(\omega_{i}^{(0)} / 3|f|\right)^{3 / 2} /\left(\omega_{i}^{(1)} R_{\max }\right)$ depends only on the Rossby number in addition to the azimuthal wavenumber $m$. The marginal stability limit can be therefore written in the general form

$$
\sqrt{\mathrm{Bu}}=\frac{1}{\sqrt{\mathrm{Ek}}} \mathscr{F}(\mathrm{Ro}, m) .
$$

This scaling is consistent with the marginal stability equation obtained by Lazar et al. (2013a) for the axisymmetric mode $(m=0)$ of the Rankine vortex equation:

$$
\sqrt{\mathrm{Bu}}=\frac{1}{\sqrt{\mathrm{Ek}}}\left(\frac{3}{8\left|a_{0}\right|}\right)^{3 / 2} \frac{(|2 \mathrm{Ro}+1|)^{7 / 4}}{|\mathrm{Ro}|}
$$

with $a_{0}=-2.33811$. In the next section (section 5), we will compare the numerical results for different baroclinic eddies to these asymptotic relations. Although they have been derived for a barotropic vortex, we will see that they work very well for baroclinic vortices.

\section{Results}

In this section, we investigate the impact of the vertical and the radial profiles of surface intensified anticyclones on the centrifugal instability depending on the Rossby and Burger numbers. We focus on the range of parameters close to the marginal stability limits. The main purpose is to build a simple stability diagram valid for various velocity profiles.

\section{a. Effects of the vertical profile}

First, we investigate the effect of the vertical profile on the centrifugal instability for submesoscale and mesoscale surface anticyclones corresponding to $\mathrm{Bu}=4$ (i.e., $R_{\max }=R_{d} / 2$ ) and $\mathrm{Bu}=1$ (i.e., $R_{\max }=R_{d}$ ) respectively. The Ekman number is kept constant with a relatively small, but not negligible value $\mathrm{Ek}=1 / 5000$. To ease the computations, the aspect ratio has been set to a moderate value: $\delta=0.5$. Nevertheless, the results would be similar for very thin oceanic eddies with $\delta \sim O\left(10^{-2}\right)$ since appendix A shows that the maximum growth rate and the most amplified vertical wavenumber of the centrifugal instability are independent of $\delta$ when $\delta<1$.

Figure 5 shows the maximum growth rate of the centrifugally unstable azimuthal modes $m=0,1,2$ as a function of the Rossby number, for submesoscale Gaussian eddies $(\mathrm{Bu}=4)$ with distinct vertical profiles. Even though their three-dimensional structures differ, the growth rates of the GG (Fig. 5b) and the GE (Fig. 5c) anticyclones exhibit similar behavior. Furthermore, a Gaussian columnar anticyclone (i.e., $\delta=\infty$ ) displays the same evolution (Fig. 5a). For this case, since $\mathrm{Bu}=\infty$ and $\mathrm{Ek}=0$, the same value of the products BuEk and $\mathrm{Ek} \delta^{2}$ as for the baroclinic anticyclones has been imposed. For all these eddies, the centrifugal instability starts slightly below $\mathrm{Ro}=-0.6$, that is, almost twice the critical value 

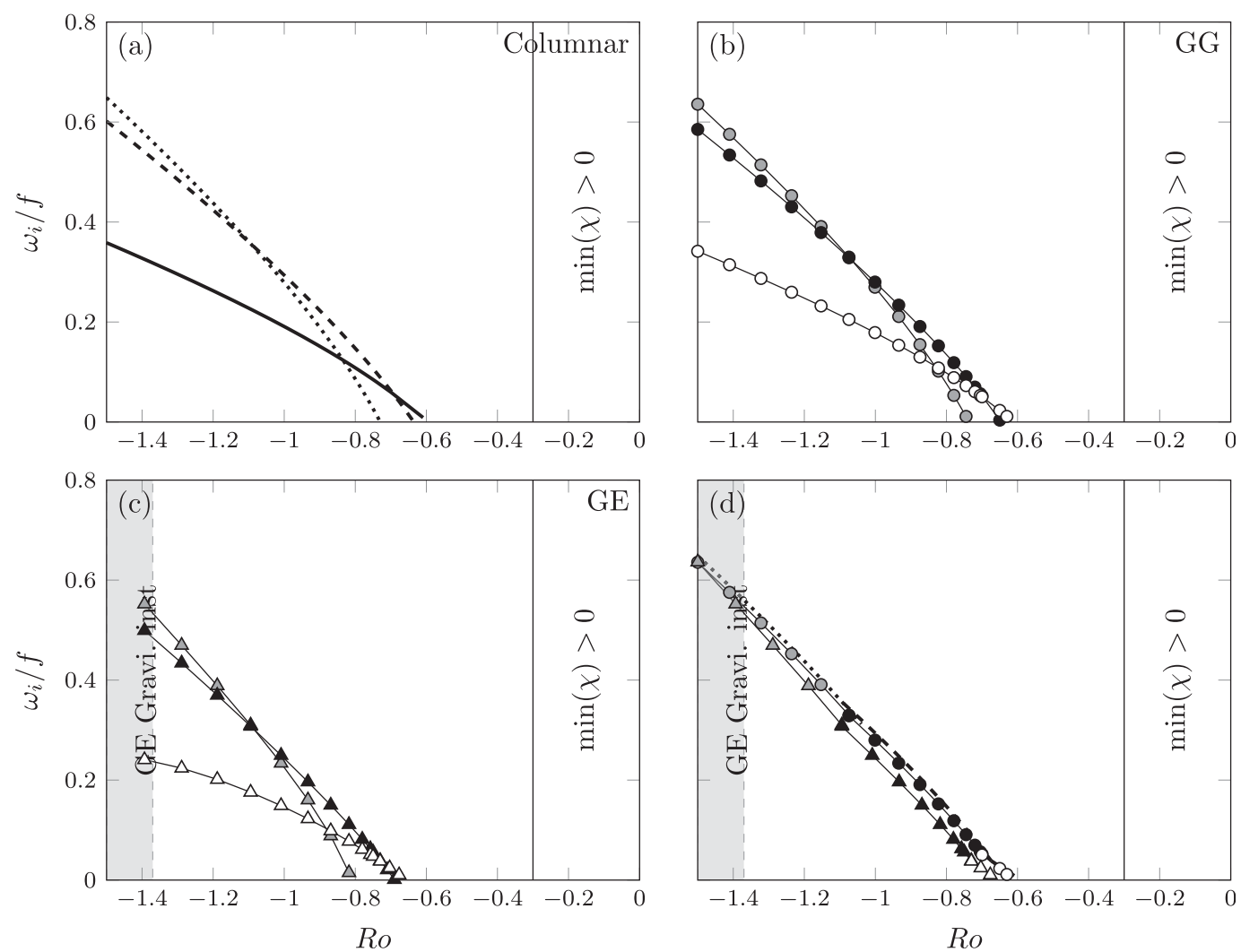

FIG. 5. Maximum growth rate $\left(\omega_{i} /|f|\right)$ as a function of Ro for different vertical profiles: (a) columnar Gaussian, (b) GG, and (c) GE eddies. In (b) and (c), the parameters are $\delta=0.5, \mathrm{Bu}=4$, and Ek $=1 / 5000$. In (a), the parameters imposed are $\mathrm{BuEk}=4 / 5000$ and $\mathrm{Ek} \delta^{2}=1 / 20000$ as for the baroclinic vortices. The lines with different symbols in (b)-(d) show the growth rate for the azimuthal wavenumbers $m=0$ (gray symbols), $m=1$ (black symbols), and $m=2$ (empty symbols). The different lines in (a) and (d) are for $m=0$ (dotted line), $m=1$ (dashed line), and $m=2$ (solid line). Vertical lines indicate the limit where $\min (\chi)=0$, i.e., the inviscid Rayleigh criterion. The gray region in (c) indicates the gravitationally unstable domain. The plot in (d) shows the maximum growth rates among all the azimuthal wavenumbers for the three profiles displayed in (a)-(c).

Ro $=-e^{1 / 2} / 2 \simeq-0.3$ predicted by the inviscid criteria in (28). As will be shown later, the difference is well accounted by the viscous criterion in (37). Close to this marginal stability limit, the most unstable mode is the azimuthal wavenumber $m=2$ for all the eddies. As $\mid$ Ro $\mid$ increases, $m=1$ becomes the most unstable mode and then the axisymmetric mode $m=0$ at higher Ro. A similar trend in the azimuthal wavenumber selection was found for columnar vortices (Billant et al. 2004), two-layer vortices (Lahaye and Zeitlin 2015), and pancake vortices (Yim and Billant 2016; Yim et al. 2016). As will be explained later, the stratification combined with viscous effects induces the dominance of the asymmetric wavenumbers $m=2$ and $m=1$ near the centrifugal instability threshold, both for barotropic and baroclinic anticyclones, even if the axisymmetric mode is the most unstable in the inviscid limit (Billant and Gallaire 2005).

To compare quantitatively the stability properties of the three eddies, their maximum growth rates are depicted, regardless of the azimuthal wavenumber, on the same graph in Fig. 5d. The growth rates of the columnar and the GG eddies are almost identical while the one associated with the GE eddy is slightly smaller but remains nevertheless very similar. This is the first evidence that for the same surface velocity profile, the vertical structure has a relatively weak impact on the growth rates of the centrifugal instability. However, if we consider other types of instability, the vertical structure is expected to have an impact. For instance, the gravitational instability threshold is located at Ro $=-1.95$ for the GG eddy and Ro $=-1.37$ for the GE eddy (Fig. 4). Moreover, the Gent-McWilliams instability (Gent and McWilliams 1986; Yim and Billant 2015; Yim et al. 2016) can also occur for the GG vortex, but the corresponding growth rates (not shown) are very small compared to the growth rate of the centrifugal instability (appendix B). 


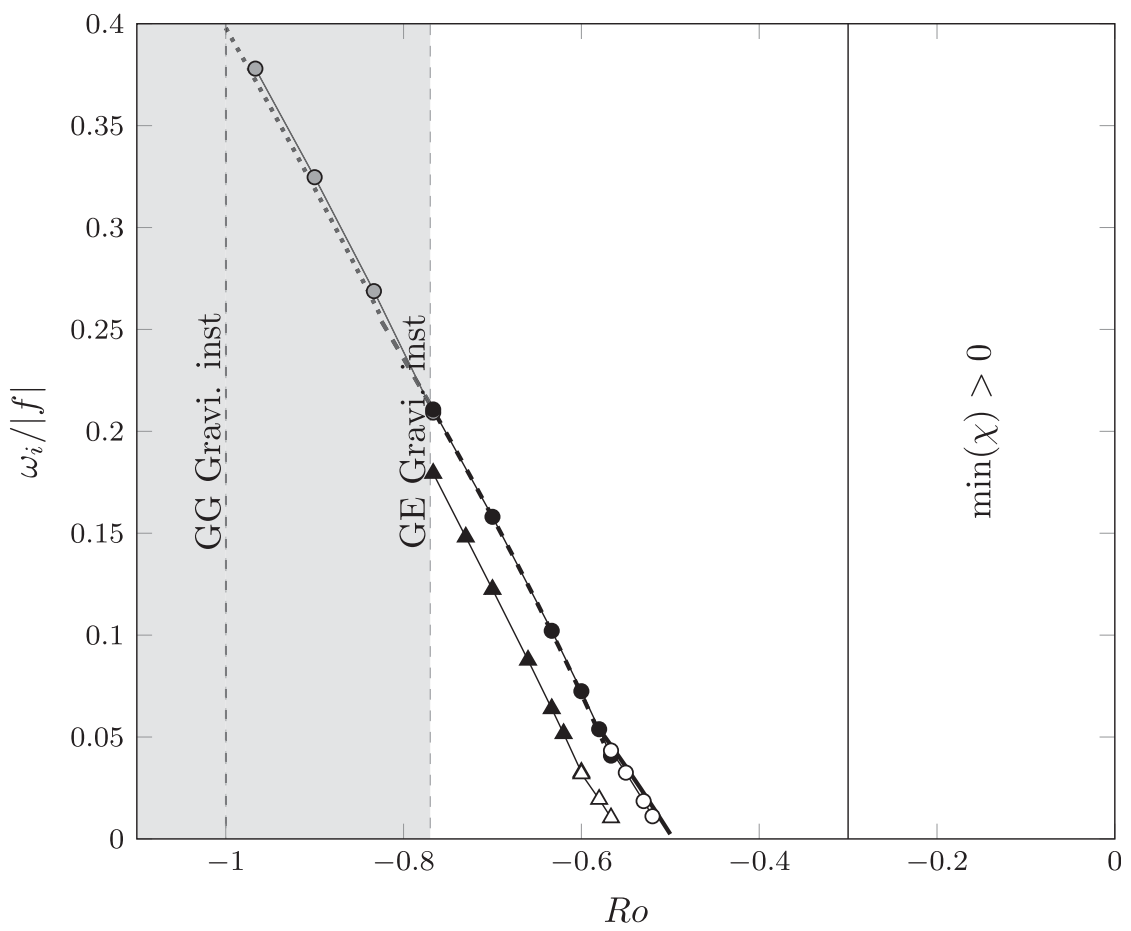

FIG. 6. As in Fig. 5d, but for $\mathrm{Bu}=1$.

Similarly, the same growth rates are displayed in Fig. 6 for $\mathrm{Bu}=1$ [i.e., mesoscale anticyclones $\left(R_{\max }=R_{d}\right)$ ] while the other parameters $(\delta, \mathrm{Ek})$ are identical. This means that only the base density $\rho_{t}$ changes (see Fig. 3 ) between Fig. 6 $(\mathrm{Bu}=1)$ and Fig. $5(\mathrm{Bu}=4)$. As for the submesoscale eddies, the first unstable modes exhibit an azimuthal wavenumber $m=2$ for these profiles. However, the centrifugal instability starts now below the threshold Ro $=$ -0.5 (more precisely, Ro $=-0.5$, Ro $=-0.52$, Ro $=$ -0.56 for the columnar, GG, and GE profiles, respectively), which is slightly higher than the marginal limit $\mathrm{Ro}=-0.6$ found for $\mathrm{Bu}=4$. This dependence with the Burger number is qualitatively consistent with the asymptotic analysis performed for columnar eddies (see section $4 \mathrm{~b})$. As for $\mathrm{Bu}=4$, the growth rates for columnar, $\mathrm{GG}$, and GE anticyclones are quite close (Fig. 6d). Again, the growth rate associated to the GE eddy is slightly below the GG and columnar eddies. This confirms that the vertical profile has a weak effect on the centrifugal instability.

In addition to the growth rates, it is interesting to examine the three-dimensional structure of the unstable modes. Figure 7 shows horizontal and vertical cross sections of the radial velocity perturbations $u_{r}$ of the most unstable eigenmode for the three different submesoscale anticyclones $(\mathrm{Bu}=4)$ for $\mathrm{Ro}=-0.7$ (i.e., close to the instability threshold). On the top view panels (Figs. 7a-c), the modes exhibit the azimuthal wavenumber $m=2$. The modes extend well outside of the regions where $\Phi<0$. In contrast, the vertical extent of the modes (Figs. 7d-f) appears to be confined within the region where $\Phi<0$. Hence, only three half wavelengths are visible in the vertical cross section for the GG and GE anticyclones (Figs. 7e,f). However, the typical vertical wavelength is very close to the one of the columnar vortex (Fig. 7d). For the smaller Rossby number Ro $=-1$ displayed in Fig. 8, the most unstable modes exhibit a $m=1$ azimuthal mode and are confined inside the $\Phi<0$ region (Fig. 8), both in the horizontal and vertical planes. The typical vertical wavelength is again similar for the three profiles. The $m=0$ mode has a similar confined structure when Ro $=-1.4$ (not shown).

Finally, Figs. $9 \mathrm{~b}$ and $9 \mathrm{~d}$ summarize the typical vertical wavenumber for the GG vortex measured directly from the vertical cross sections of the eigenmodes for different Rossby numbers. The lines show also the asymptotic prediction in (35) for columnar vortices. They are in very good agreement except for the axisymmetric mode. We can remark that the vertical wavelength depends weakly on the Rossby number. As shown by (35), it is mainly controlled by the Burger and Ekman numbers. The growth rates for the GG vortex are also in good agreement with the asymptotic prediction in (36) (Figs. 9a,c).

\section{b. Effects of the radial profile}

In the previous section, the radial profile has been fixed to the Gaussian angular velocity profile. However, 

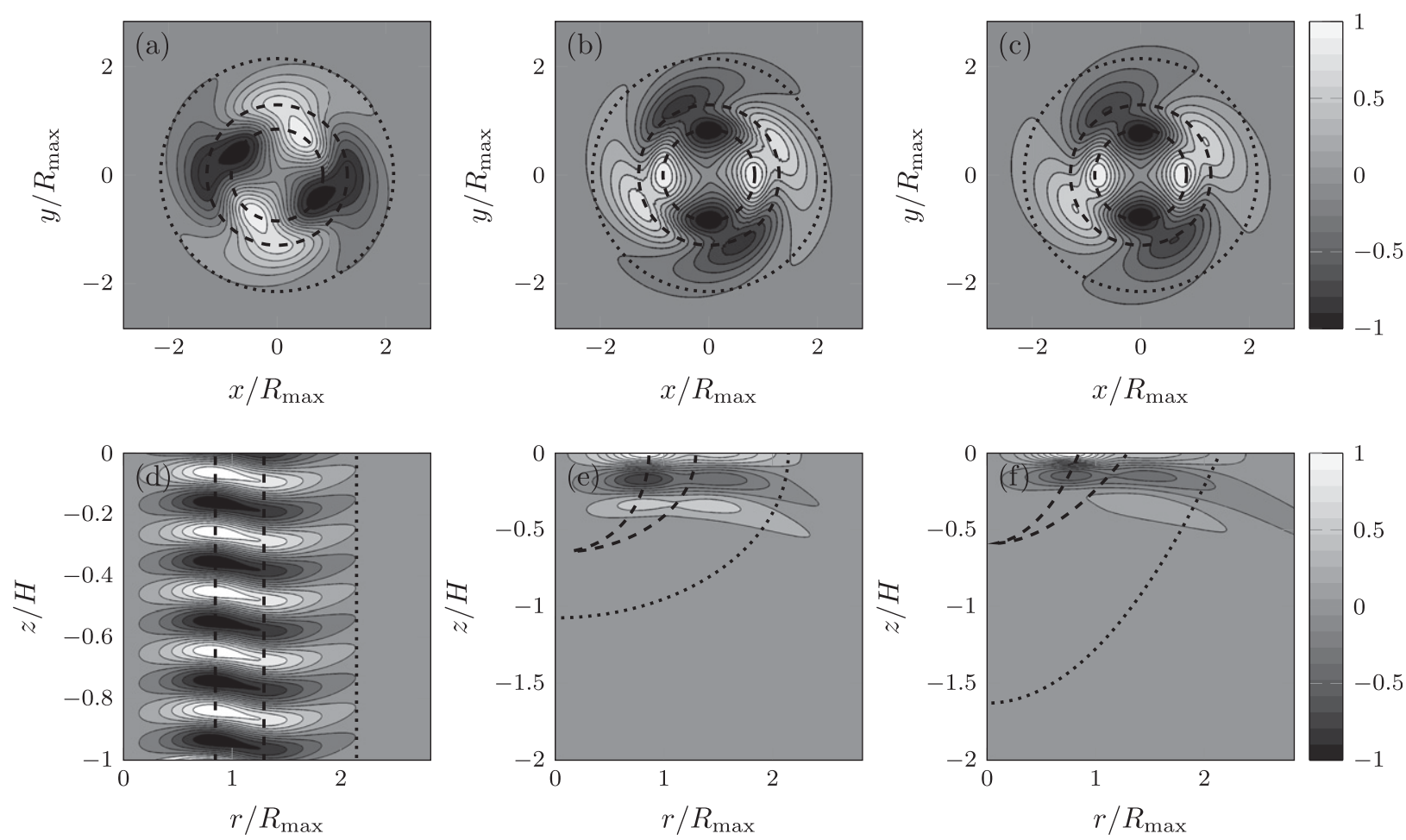

FIG. 7. (a)-(c) Horizontal cross sections at $z=0$ and (d)-(f) vertical cross sections (only for $r>0$ ) of the real part of the normalized radial velocity perturbation $\left[\operatorname{Re}\left(u_{r}\right) / \max \left(\left|u_{r}\right|\right)\right]$ for (left) columnar, (center) GG, and (right) GE vortices for $m=2$ and Ro $=-0.7$. The other parameters are the same as in Fig. 5: $\mathrm{Bu}=4, \mathrm{Ek}=1 / 5000, \delta=0.5$ for surface intensified vortices, and $\mathrm{BuEk}=4 / 5000$ and $\mathrm{Ek} \delta^{2}=4 / 20000$ for the columnar vortex. The dotted lines indicate the limit of the base vortex where $\Omega=0.1 \Omega_{0}$. The dashed lines represent the contours where the generalized Rayleigh discriminant $\Phi$ vanishes.

such velocity profile is not universal and oceanic eddies exhibit a wide variety of profiles with a steepness parameter ranging from $\alpha=1.5$ to $\alpha=3$ (Ioannou et al. 2017). Therefore, we investigate in this section the effect of the radial angular velocity profile on the centrifugal instability. The steepness parameter is varied from $\alpha=1.5$ (G1.5G) to $\alpha=3$ (G3G) while keeping a Gaussian vertical angular velocity profile: $G(\hat{z})=e^{-\hat{z}^{2} / 2}$. We recall that the inviscid threshold for the centrifugal stability depends on the steepness parameter $\alpha$ : Ro $>$ $\mathrm{Ro}_{c \infty}=-1 / 2 e^{-1 / \alpha}$. However, the recent stability analysis of Lazar et al. (2013a) has shown that when the viscous dissipation is taken into account, the critical Rossby number for various profiles of columnar vortices are very close. Here, we will determine if this is also the case for baroclinic surface intensified anticyclones.

Figure 10 shows the evolution of the growth rate of the most unstable centrifugal mode as a function of the vortex intensity for the three distinct velocity profiles: GG $(\alpha=2), \mathrm{G} 1.5 \mathrm{G}$, and G3G for $\mathrm{Bu}=4$ (Figs. 10a,b) and $\mathrm{Bu}=1$ (Figs. 10c,d). We use both the vortex Rossby number Ro (left panels) and the normalized core vorticity $\zeta_{z}(0) / f$ (right panels) to quantify the vortex intensity. The growth rate for the three velocity profiles are well separated when represented as a function of the normalized core vorticity while they almost collapse when plotted as a function of the vortex Rossby number. Indeed, for submesoscale eddies, that is, $\mathrm{Bu}=4$ (Fig. 10a), the marginal stability limit is crossed for the same value of the Rossby number $\mathrm{Ro} \simeq-0.62 \pm 0.02$ for the three eddies, while the critical values of the normalized core vorticity differ (Fig. 10b). For larger eddies, that is, mesoscale anticyclones with $\mathrm{Bu}=1$ (lower panels), the critical Rossby numbers are slightly more dispersed $(\mathrm{Ro} \simeq-0.5 \pm 0.05)$ but nevertheless the growth rate curves for the three eddies in Fig. 10c are closer than in Fig. 10d. Hence, the centrifugal instability of baroclinic anticyclones is also controlled by the vortex Rossby number as found by Lazar et al. (2013a) for barotropic anticyclones.

The present study focuses on the centrifugal instability but it should be recalled that other types of instability can exist as mentioned in the introduction and section 2. These different instabilities are studied in appendix B and shown to be dominant only for parameters where the centrifugal instability is stable or weakly 

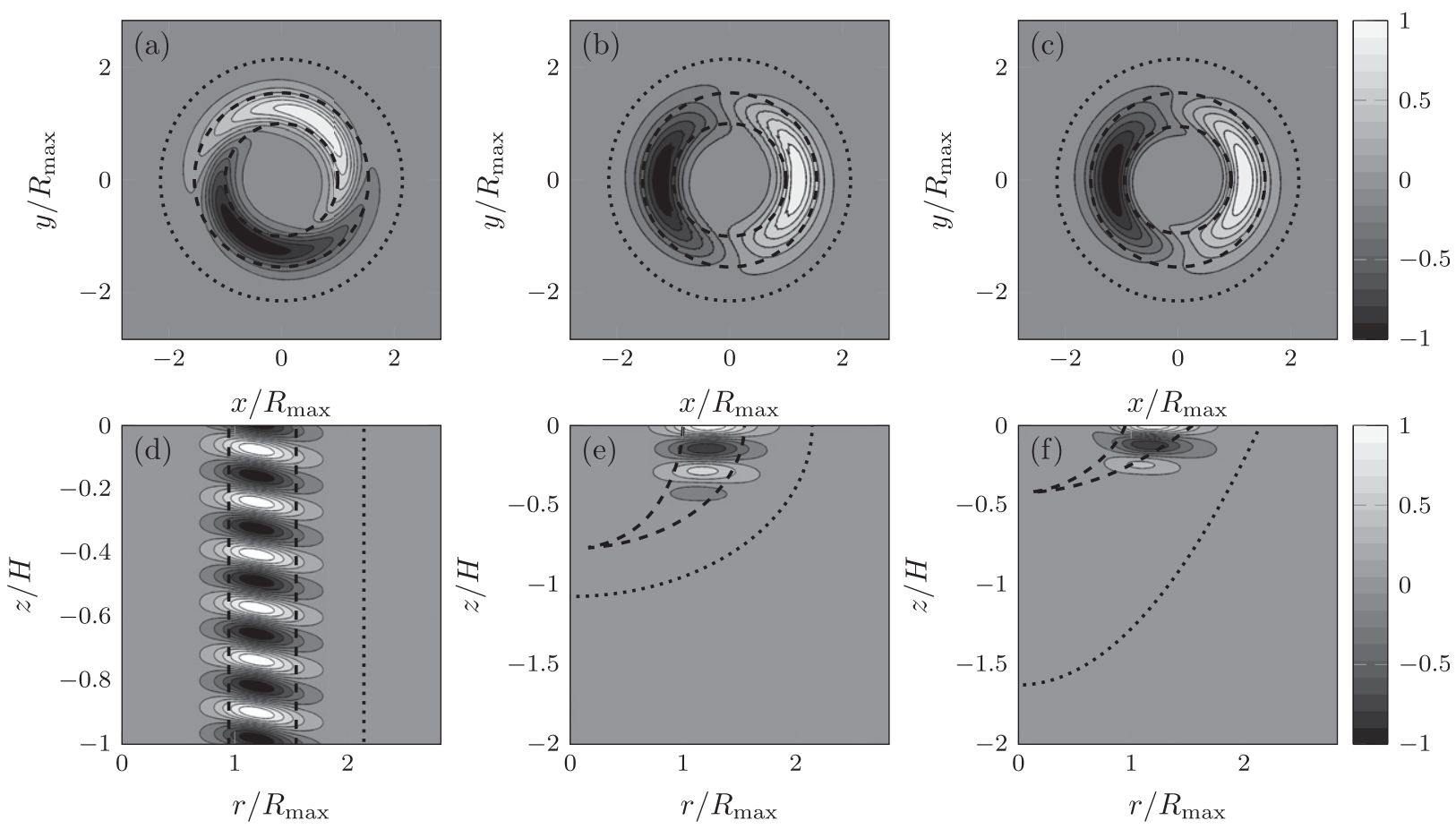

FIG. 8. As in Fig. 7, but for $m=1$ and Ro $=-1$.

unstable. For example, for the profile $\mathrm{G} 3 \mathrm{G}$ for $\mathrm{Bu}=4$, the centrifugal instability is dominant when $\mathrm{Ro}<-0.73$, that is, slightly below the marginal Rossby number found previously, $\mathrm{Ro} \simeq-0.6$. When Ro $>-0.73$, the $m=2$ baroclinic-shear instability is dominant.

\section{c. Stability diagrams}

Following Lazar et al. (2013a), who provided the first stability diagrams for circular barotropic anticyclones in the $(\sqrt{\mathrm{Bu}}, \mathrm{Ro})$ parameter space, similar diagrams have been established for the various baroclinic surface anticyclones studied in the previous sections. Unlike Lazar et al. (2013a), the azimuthal wavenumber has not been restricted to $m=0$, but $m=1, m=2$, and $m=3$ have been also considered.

Figure 11 shows the marginal stability limits for different anticyclonic vortices as function of their relative size $\sqrt{\mathrm{Bu}}=R_{d} / R_{\max }$ and intensity Ro. These stability diagrams are plotted for two distinct Ekman numbers: $\mathrm{Ek}=1 / 2500$ (Figs. 11a,c) and Ek =1/5000 (Figs. 11b,d) for different vertical profiles (Figs. 11a,b) and radial profiles (Figs. 11c,d). The vertical lines correspond to the inviscid stability limit $\mathrm{Ro}_{c \infty}=-1 /\left(2 e^{1 / \alpha}\right)$ for $\alpha=1.5$ (dotted line), $\alpha=2$ (solid line), and $\alpha=3$ (dashed line). The limits of the gravitational instability for the different profiles are also indicated by gray curves in the bottom part of the plots. For all the vortices, the viscous dissipation combined to the vertical stratification increases significantly the stable area of the centrifugal instability (located below the marginal stability curves) when the Burger number increases. The gravitational instability (GI) affects mainly large eddies having a characteristic radius $R_{\max }$ roughly equal or larger than the local deformation radius $R_{d}$.

The upper panels (Figs. 11a,b) compare the marginal stability limits of the columnar Gaussian eddy with the baroclinic GG and GE eddies regardless of the azimuthal wavenumber. In agreement with Figs. 5d and 6, the marginal stability limits of the baroclinic GG (crosses) and barotropic columnar (solid line) anticyclones almost coincide while the one associated with the GE anticyclone (triangles) is slightly below. Hence, the vertical structure of the surface intensified anticyclones has a very weak impact on the threshold of the centrifugal instability and has roughly the same marginal stability limit as barotropic anticyclones. This strongly suggests that the asymptotic analysis, which is strictly valid only for columnar eddies, can also provide meaningful results for baroclinic vortices. Indeed, the asymptotic stability limit in (37) (dashed lines) matches almost perfectly the stability limit obtained numerically when the most unstable mode corresponds to the azimuthal wavenumber $m=2$ (black curves) but starts to deviate for $m=1$ (gray curves) when the Burger number increases. Similar conclusions can be drawn from Figs. 11c and 11d where the marginal limits of the 

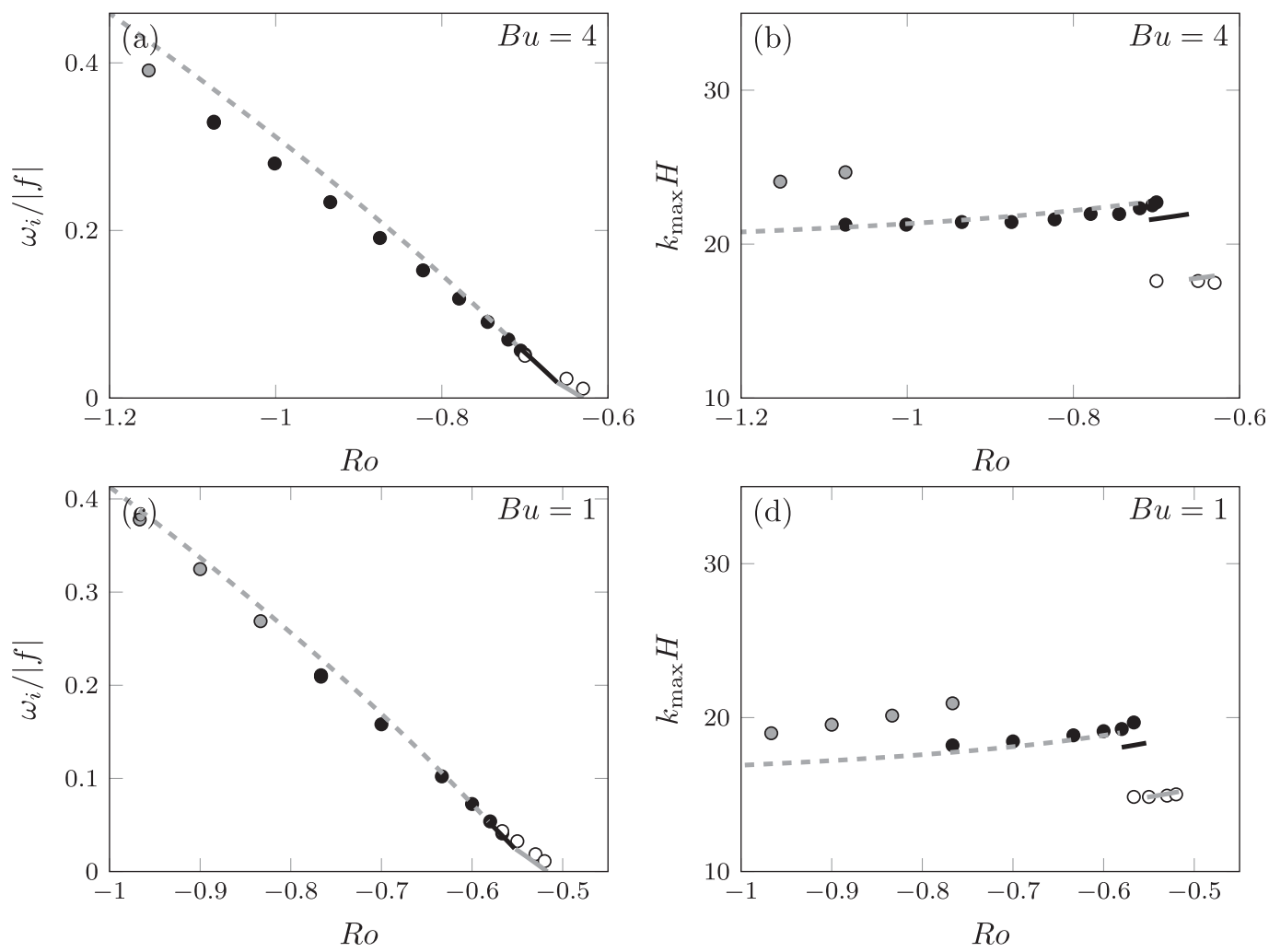

FIG. 9. Comparison between (left) the maximum growth rates and (right) the most amplified vertical wavenumber $k_{\max } H$ predicted by (36) and (35) (lines) and obtained numerically for the GG eddy (symbols) for (a), (b) $\mathrm{Bu}=4$ and (c),(d) $\mathrm{Bu}=1$ for $\mathrm{Ek}=1 / 5000$ and $\delta=0.5$. The different symbols and lines correspond to the azimuthal wavenumber: $m=0$ (gray circles and dashed line), $m=1$ (black circles and solid line), and $m=2$ (empty circles and gray solid line).

centrifugal instability obtained numerically and asymptotically are plotted for distinct radial profiles (G1.5G and G3G).

As in Lazar et al. (2013a), our results reveal that the stability diagrams for various type of anticyclones having different horizontal and/or vertical structure are almost identical in the Rossby and Burger parameter space. When the eddy is baroclinic, these nondimensional numbers should be evaluated at the level where the vortex is the most intense. For the present surface intensified anticyclones, the maximum azimuthal velocity $V_{\max }$ and the corresponding radius $R_{\max }$, which are needed to estimate the vortex Rossby and the Burger numbers, should then be evaluated at the surface $(z=0)$. Unlike Lazar et al. (2013a), who considered only the axisymmetric mode $(m=0)$, we have found that all the marginal centrifugal modes are asymmetric and correspond to the modes $m=2$ when $\sqrt{\mathrm{Bu}} \leqslant 4$ and $m=1$ for higher Bu. Hence, we could expect some discrepancies between the asymptotic stability criterion (39) of Lazar et al. (2013b) and the marginal stability curves obtained numerically. Figure 12 shows indeed that the asymptotic stability criterion (39) (black dashdotted line) underestimates the CI area. The asymmetric modes appear to be unstable for weaker Rossby numbers than the axisymmetric modes regardless of the Burger number.

\section{d. Asymptotic marginal stability criterion}

The good agreement between the asymptotic criterion (37) and the numerical results in Fig. 11 has pushed us to conduct further the asymptotic analysis of section $4 \mathrm{~b}$. Using additional assumptions, we can indeed further simplify the formula (37) in order to obtain an explicit criterion that will be easier to use.

When $\mathrm{Ro}=\mathrm{Ro}_{c \infty}$, the radius $r_{0}$ satisfying (33) vanishes. Therefore, when the Rossby number is close to $\mathrm{Ro}_{c \infty}$, we can find $r_{0}$ by expanding it in terms of $1-\mathrm{Ro}_{c^{\infty}} / \mathrm{Ro}$. More precisely, it is convenient to expand $r_{0}^{\alpha} / \alpha$ as

$$
\frac{r_{0}^{\alpha}}{\alpha}=a_{1}\left(1-\frac{\mathrm{Ro}_{c \infty}}{\mathrm{Ro}}\right)+O\left(1-\frac{\mathrm{Ro}_{c \infty}}{\mathrm{Ro}}\right)^{2}
$$



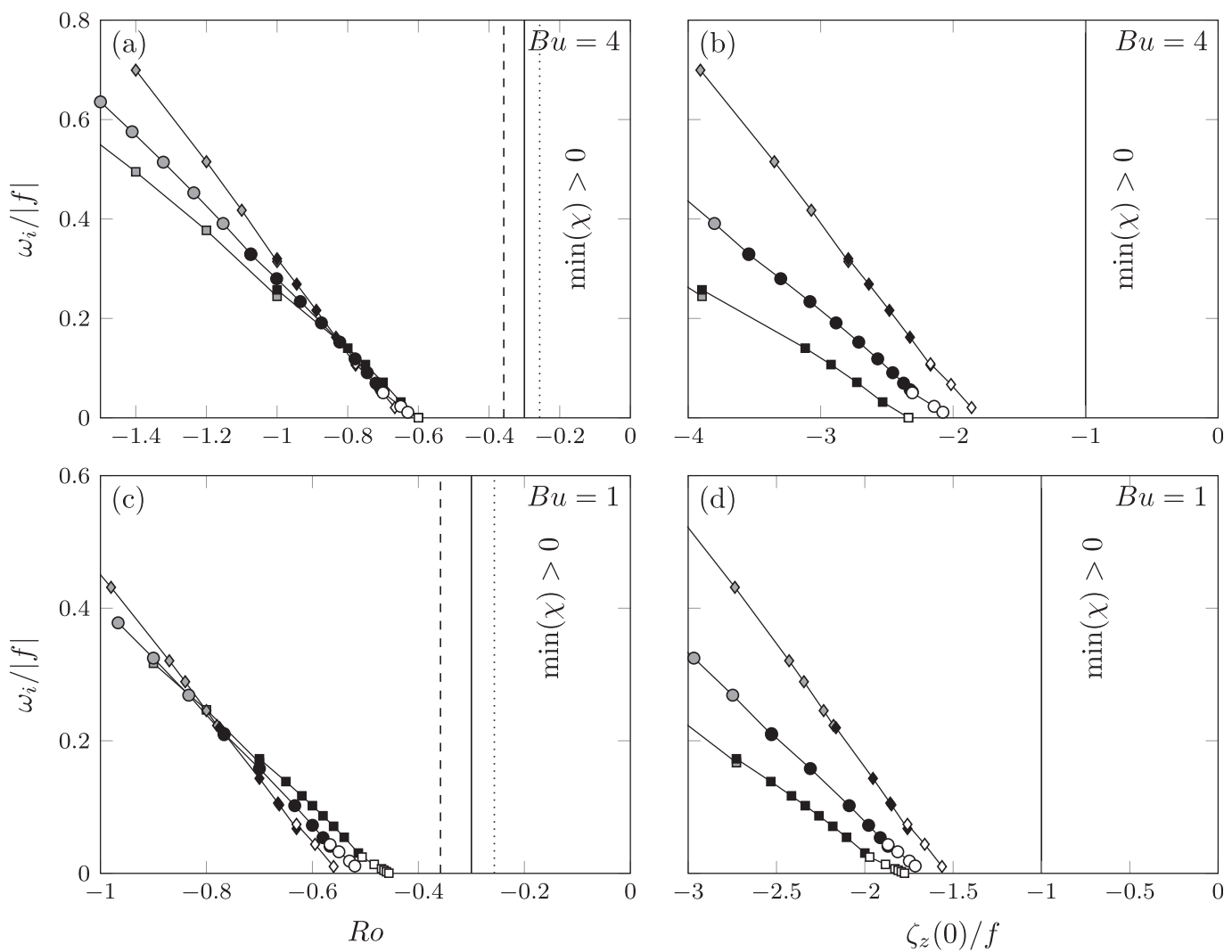

FIG. 10. Maximum growth rate for different radial velocity profiles at $E k=1 / 5000$ as a function of (left) Ro and (right) $\zeta_{z}(0) / f$ for (a),(b) $\mathrm{Bu}=4$ and (c),(d) $\mathrm{Bu}=1$. The different symbols correspond to the profiles: $\mathrm{GG}$ (circle), G3G (diamond), and G1.5G (square) and the different azimuthal wavenumbers: $m=0$ (gray symbols), $m=1$ (black symbols), and $m=2$ (empty symbols). Vertical lines indicate the limit $\min (\chi)=0$, i.e., the inviscid Rayleigh criterion. In (a) and (c), the threshold $\min (\chi)=0$ varies with the steepness parameter $\alpha: \alpha=1.5$ (dotted line), $\alpha=2$ (solid line), and $\alpha=3$ (dashed line).

since $r_{0}$ appears always under this form in (32). The coefficient $a_{1}$ is given in appendix C. Using (40), (30) and (31) become

$\omega_{i}^{(0)}=\Omega_{0}\left[c_{1}\left(1-\frac{\mathrm{Ro}_{c^{\infty}}}{\mathrm{Ro}}\right)+O\left(1-\frac{\mathrm{Ro}_{c^{\infty}}}{\mathrm{Ro}}\right)^{2}\right]$,

$\omega_{i}^{(1)}=\frac{1}{R_{\max }}\left[c_{3}\left(1-\frac{\mathrm{Ro}_{c^{\infty}}}{\mathrm{Ro}}\right)^{-1 / \alpha}+O\left(1-\frac{\mathrm{Ro}_{c^{\infty}}}{\mathrm{Ro}}\right)^{1-(1 / \alpha)}\right]$,

where $c_{1}$ and $c_{3}$ depend only on $m$ and $\alpha$. Their expressions are detailed in appendix C. Note that the stratification has been assumed to be strong in (31) so that $N^{2} \gg \chi\left(r_{0}\right)$. Using (41) and (42), the criterion (37) reads

$$
\sqrt{\mathrm{Bu}}=\frac{c_{4}}{\sqrt{\mathrm{Ek}}} \frac{\left|\mathrm{Ro}-\mathrm{Ro}_{c^{\infty}}\right|^{3 / 2+1 / \alpha}}{|\mathrm{Ro}|^{1 / \alpha}},
$$

where $c_{4}=\left[c_{1} /\left(3 e^{-1 / \alpha}\right)\right]^{3 / 2}\left(2 / c_{3}\right)$. For $m=2$, the coefficient $c_{4}$ simplifies to

$$
c_{4}=\frac{-\frac{\alpha}{5}+\sqrt{\alpha}-\frac{2}{3}}{9\left(\frac{1}{2} e^{-1 / \alpha}\right)^{3 / 2}},
$$

giving $c_{4}=0.23$ for $\alpha=2$. The simplified criterion (43) with $m=2$ and $\alpha=2$ is represented by a thick dashed line in Fig. 12. It is in very good agreement with the marginal limits obtained numerically for all the profiles investigated (i.e., not only for $\alpha=2$ ). The coefficient $c_{4}$ is indeed weakly dependent on $\alpha$ and varies from $c_{4}=0.22$ to $c_{4}=0.24$ when $\alpha$ goes from $\alpha=1.5$ to $\alpha=3$. In addition, $c_{4}$ has also a similar value for $m=1$ and $\alpha=2$ : $c_{4}=0.2$. Hence, we suggest to use the asymptotic equation (43) for the marginal stability limit with $c_{4}=$ 0.23 when the oceanic eddy profile is not precisely known. This criterion is more accurate than the criterion 

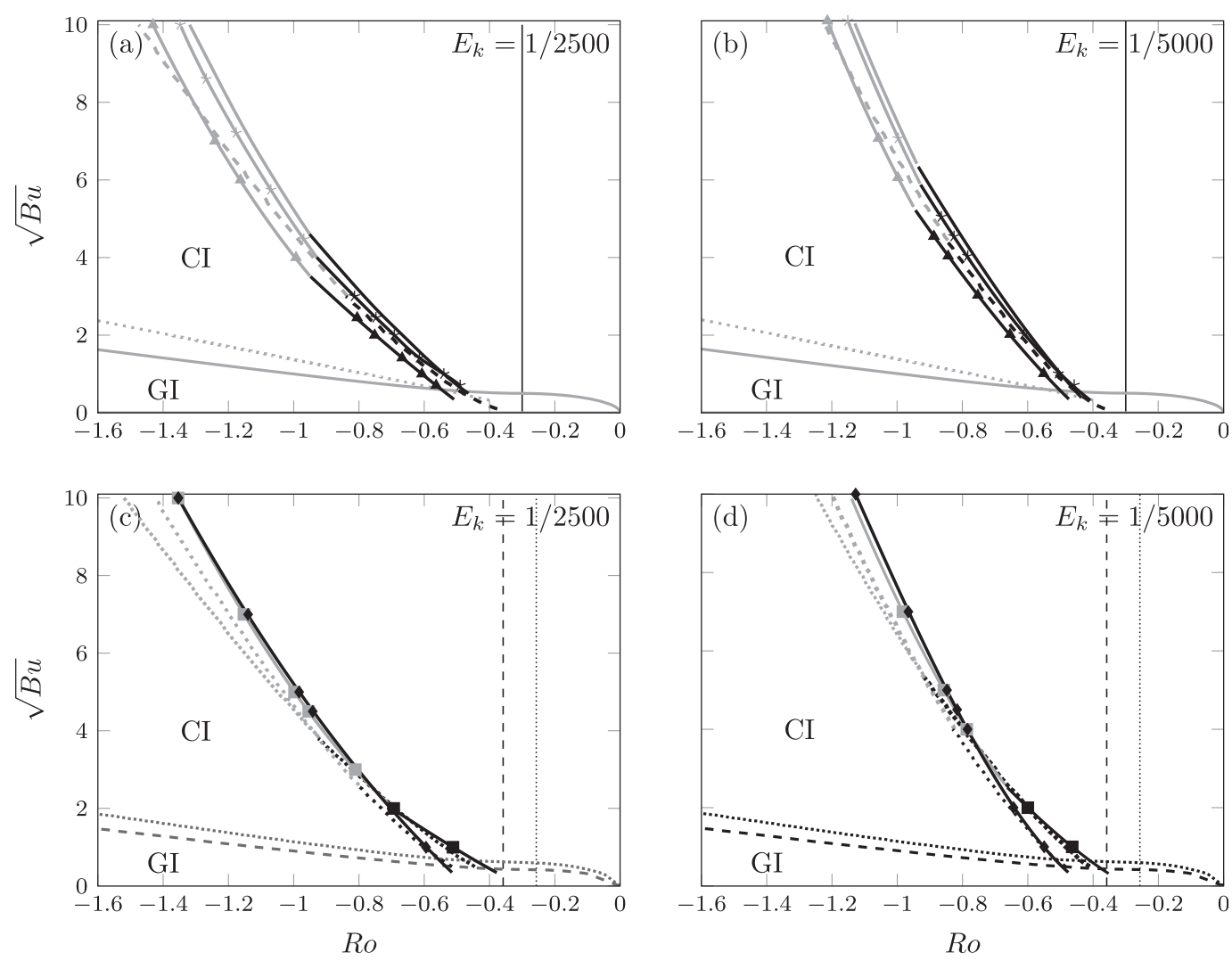

FIG. 11. Marginal stability lines in the parameter space $(\mathrm{Ro}, \sqrt{\mathrm{Bu}})$ for different vertical profiles: columnar (lines without symbols), GG (stars), GE (triangles) eddies and predicted by the asymptotic formula (37) (dashed lines), for (a) $E k=1 / 2500$ and (b) $E k=1 / 5000$. (c),(d) As in (a) and (b), but for different radial profiles: G3G (diamonds) and G1.5G (squares) eddies and asymptotic results (fine dotted lines for G1.5G; coarse dotted lines for G3G). The color indicates the dominant azimuthal wavenumber: $m=1$ (gray) and $m=2$ (black). Gray lines at the bottom of the figures indicate the limits of the gravitational instability for the GG (solid), GE (dotted), G3G (dashed), and G1.5G (fine dotted) profiles. The corresponding vertical lines show the inviscid Rayleigh criterion $\min (\chi)=0$.

(39) obtained by Lazar et al. (2013a) for axisymmetric modes (dash-dotted line in Fig. 12). Equations (43) and (39) look indeed similar but the power exponents differ.

The effect of the dissipation is illustrated in Fig. 13 where the asymptotic stability limits (43) with $c_{4}=0.23$ for $\alpha=2, m=2$ are plotted for a wide range of Ekman numbers, from $\mathrm{Ek}=1 / 2500$ to $\mathrm{Ek}=10^{-5}$. The viscous marginal stability limits (43) and (39) become closer to the inviscid limit $\mathrm{Ro}_{c} \simeq-0.3$ for $\alpha=2$ when the Burger number tends to zero. However, a significant shift remains between these two curves for finite Burger number even for a very weak dissipation $\left(\mathrm{Ek}=10^{-5}\right)$. The simplified criterion (43) is very close to the full asymptotic criterion (37) except when $|\mathrm{Ro}| \geq-0.6$ since the assumption $\left|\mathrm{Ro}-\mathrm{Ro}_{c_{\infty}}\right| \ll 1$ becomes less valid.

The simplified expressions (41) and (42) offer also the possibility to explain simply the dominance of the asymmetric centrifugal modes close to the marginal limit. Using (41) and (42), the maximum growth rate (36) becomes

$$
\begin{aligned}
\frac{\omega_{i \max }}{|f|}= & |\operatorname{Ro}| e^{1 / \alpha}\left[c_{1}\left(1-\frac{\mathrm{Ro}_{c_{\infty}}}{\mathrm{Ro}}\right)\right] \\
& -3(\mathrm{BuEk})^{1 / 3}\left[\frac{c_{3}}{2}\left(1-\frac{\mathrm{Ro}_{c_{\infty}}}{\mathrm{Ro}}\right)^{-1 / \alpha}\right]^{2 / 3} .
\end{aligned}
$$

The first term on the rhs of (45) is the maximum growth rate in the inviscid limit while the second term represents the damping effect of the stratification and viscous dissipation. These terms depend on the azimuthal wavenumber $m$ only through the constants $c_{1}$ and $c_{3}$. If we consider the case $\alpha=2$, we have $c_{1}=0.707$ for $m=0$, $c_{1}=0.66$ for $m=1$, and $c_{1}=0.5$ for $m=2$. Thus, $c_{1}$ decreases with $m$, meaning that the growth rate (45) in the inviscid limit is maximum for $m=0$. However, the constant $c_{3}$ is $c_{3}=2.45$ for $m=0, c_{3}=2.14$ for $m=1$, and $c_{3}=1.14$ for $m=2$. Therefore, the damping effect decreases with $m$ for a given Rossby number. In addition, this damping term increases when $\mathrm{Ro} \rightarrow \mathrm{Ro}_{c \infty}$ while the 


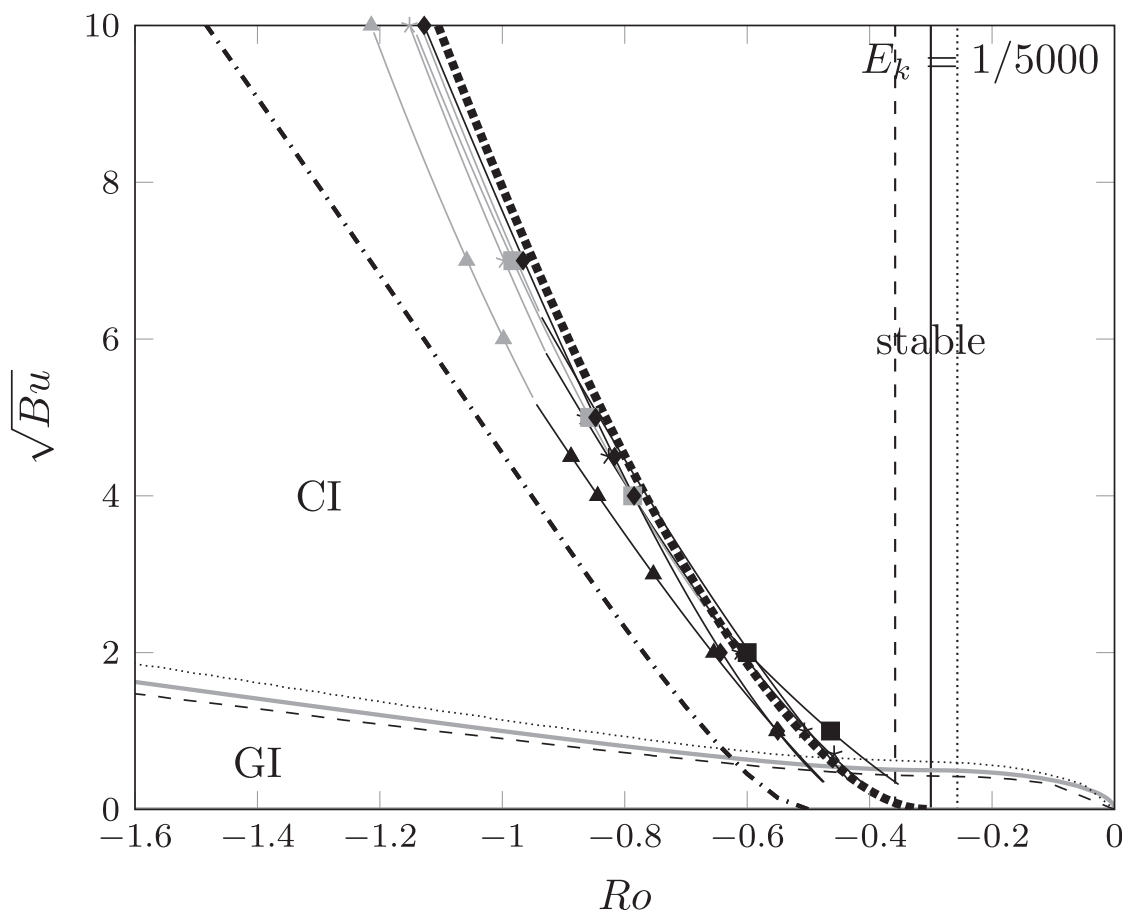

FIG. 12. All the marginal stability lines of Figs. 11b and 11d for different profiles plotted together and compared to the simplified asymptotic condition (43) with $\alpha=2, m=2$ (thick dashed line) and to the condition (39) of Lazar et al. (2013a) (dash-dotted line).

first term of (45) decreases. Hence, the asymmetric centrifugal modes can become the most unstable near the marginal limit since they are less damped than the axisymmetric mode. Physically, it is likely that the axisymmetric mode is more damped than asymmetric ones because it involves only radial and vertical motions. The latter motions are indeed inhibited by the stratification. In contrast, the nonaxisymmetric modes can still exist even if the vertical motions are weak since they involve also azimuthal motions.

\section{Summary and discussion}

We have investigated the linear stability of baroclinic surface intensified anticyclones to three-dimensional perturbations for arbitrary azimuthal wavenumbers. The main purpose is to build a stability diagram for the centrifugal (inertial) instability and derive a simple stability criterion taking into account the stratification and the dissipation more suitable for oceanic eddies than the standard inviscid Rayleigh criterion.

We have first performed a numerical stability analysis by means of a finite element method $($ FreeFem ++ ) that solves the full three-dimensional eigenvalue problem for various steady and circular baroclinic anticyclones located at the ocean surface. As shown by Nguyen et al.
(2012), Yim et al. (2016), and Mahdinia et al. (2017), this work goes beyond the barotropic idealization that was often previously used for stability analyses of stratifiedrotating vortices (Kloosterziel et al. 2007; Lazar et al. 2013a). The results reveal that the growth rates and the marginal stability limit of the centrifugal modes are close to those calculated for an equivalent barotropic columnar eddy. In other words, the vertical velocity profiles of the oceanic eddies do not play an important role in the centrifugal instability. The vertical structure affects only the vertical extent of the eigenmode. Hence, most of the results obtained for idealized columnar vortices, especially asymptotic results, can be applied to realistic baroclinic anticyclones. In addition, we have found that the centrifugal modes exhibit an asymmetric azimuthal wavenumber ( $m=2$ or $m=1)$ close to the marginal stability limit for all the eddies regardless of their vertical structure. Even if they are less unstable than the axisymmetric mode in the inviscid limit, the asymmetric centrifugal modes can become dominant because of their weaker sensitivity to the damping effect of the stratification and viscous dissipation. This has been also found by Lahaye and Zeitlin (2015) for idealized two-layer vortices and Billant et al. (2004) for stratified rotating vortices, and by Yim and Billant (2016); Yim et al. (2016) for pancake vortices. Hence, 


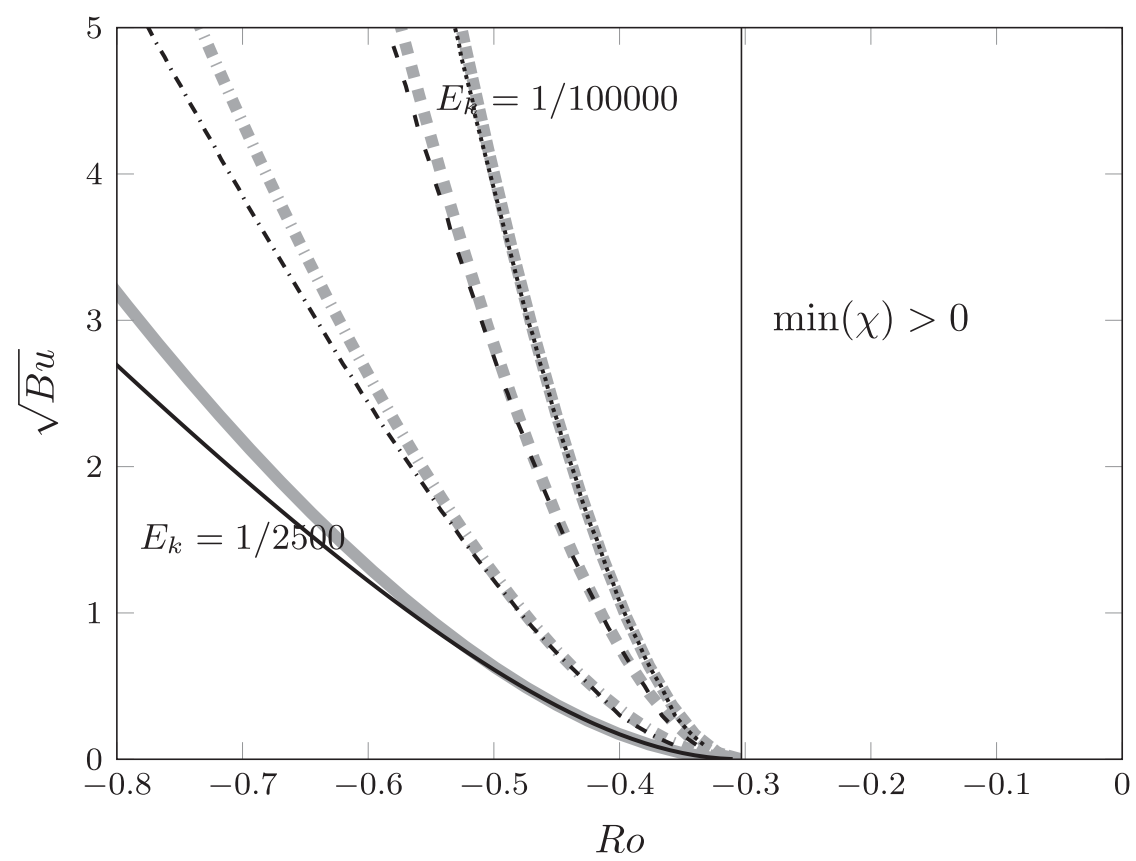

FIG. 13. Asymptotic marginal stability limits (37) (black lines) and (43) for $\alpha=2, m=2$ (thick gray lines) for different Ekman numbers: $E k=1 / 2500$ (solid line), Ek =1/10 000 (dashdotted line), $\mathrm{Ek}=1 / 50000$ (dashed line), and $\mathrm{Ek}=1 / 100000$ (dotted line).

the stability diagram proposed by Lazar et al. (2013a) for axisymmetric $(m=0)$ perturbations on intense circular anticyclones has to be extended to asymmetric perturbations.

Therefore, in a second step, we have used the asymptotic analysis proposed by Yim and Billant (2016) for pancake eddies to obtain the simplified marginal stability criterion (43) for the azimuthal wavenumber $m=2$, which depends on four dimensionless parameters: the Rossby number $\mathrm{Ro}=V_{\max } /\left(f R_{\max }\right)$, the Burger number $\mathrm{Bu}=N_{0}^{2} H^{2} /\left(f R_{\max }\right)^{2}=\left(R_{d} / R_{\max }\right)^{2}$, the Ekman number $\mathrm{Ek}=\nu /\left(f H^{2}\right)$, and the steepness parameter $\alpha$ of the radial profiles of azimuthal velocity $V_{\theta}(r)=V_{\max } e^{1 / \alpha}\left(r / R_{\max }\right) \exp \left[-\left(r / R_{\max }\right)^{\alpha} / \alpha\right]$. The recent analysis of Ioannou et al. (2017) of mesoscale eddies has shown that $\alpha$ is distributed between 1.5 and 3 with a mean value around $\alpha=2$ (i.e., the Gaussian profile). For such range, the asymptotic stability criterion (43) depends weakly on $\alpha$. Lazar et al. (2013a) have also found that the impact of the radial velocity profile on the centrifugal instability limit is weak when the dissipation and the background stratification are not too small. Therefore, we suggest using the asymptotic criterion (43) with $\alpha=2$ as a generalized stability criterion for most of the oceanic anticyclones. This instability criterion depends only on three dimensionless parameters (Ro, $\mathrm{Bu}$, and $\mathrm{Ek})$ and can be written as follows:

$$
\sqrt{\mathrm{Bu}}=\frac{R_{d}}{R_{\max }} \leq \frac{0.23}{\sqrt{\mathrm{Ek}}} \frac{(\mathrm{Ro}+0.3)^{2}}{\sqrt{|\mathrm{Ro}|}}
$$

It takes into account the stratification and the dissipation through $\mathrm{Bu}$ and Ek, respectively. According to this criterion, baroclinic anticyclones can remain stable even if they have a core region of negative absolute vorticity [i.e., $\zeta(0)<-f$ or equivalently Ro $<-0.3$ ], provided that they are small enough. In other words, the stability domain of submesoscale eddies in the parameter space $(\mathrm{Ro}, \mathrm{Bu})$ is extended in comparison with the standard Rayleigh criterion: Ro $<-0.3$.

The main advantage of the above asymptotic criterion is that both the Rossby and the Burger numbers can be easily estimated in the ocean. Indeed, for a quasi-circular eddy, the maximal velocity $V_{\max }$ and the corresponding speed radius $R_{\max }$ can be obtained directly from in situ vessel-mounted acoustic Doppler current profiler (VMADCP) high-frequency radar (HFR) current measurements or from altimetric measurements that estimate the surface velocity. The local deformation radius $R_{d}$ can be also easily estimated from a single density profile. On the other hand, estimating the Ekman number Ek is not straightforward since we should consider the vertical turbulent eddy viscosity instead of the molecular viscosity. We can assume as a first approximation that such turbulent 
TABLE 1. Typical dynamical values of few intense anticyclones obtained from in situ measurements. These values were calculated from Fig. 6 of Chavanne et al. (2010) and Table 1 of Bosse et al. (2016).

\begin{tabular}{lccccc}
\hline \hline \multicolumn{1}{c}{ Name } & Date & $R_{\max }(\mathrm{km})$ & $V_{\max }\left(\mathrm{cm} \mathrm{s}^{-1}\right)$ & $|\mathrm{Ro}|=V_{\max } /\left(f R_{\max }\right)$ & $\sqrt{\mathrm{Bu}=R_{\max } / R_{d}}$ \\
\hline Oahu & Oct 2002 & 15 & 35 & 0.4 & 1.7 \\
SCV North Med 1 & Feb 2011 & 6.5 & 19.6 & 0.31 & 1.34 \\
SCV North Med 2 & Jun 2012 & 6.5 & 17.4 & 0.27 & 1.7 \\
SCV North Med 3 & Feb 2013 & 4.1 & 18.4 & 0.45 & 2.0 \\
SCV North Med 4 & May 2013 & 3.8 & 12.4 & 0.34 & 0.98 \\
\hline
\end{tabular}

eddy viscosity is of the same order of magnitude than the turbulent diapycnal mixing. However, the latter quantity may vary by several orders of magnitude: from $\kappa_{z}=10^{-5} \mathrm{~m}^{2} \mathrm{~s}^{-1}$ in the deep and stratified ocean (Ledwell et al. 1998) to $\kappa_{z}=10^{-3} \mathrm{~m}^{2} \mathrm{~s}^{-1}$ in surface oceanic fronts (Thompson et al. 2007) or above topography (Kunze and Toole 1997; Garabato et al. 2004). If we use an intermediate diapycnal diffusivity of $\kappa_{z} \sim \nu \sim 10^{-4} \mathrm{~m}^{2} \mathrm{~s}^{-1}$ for a surface eddy with a characteristic depth of $H \sim 100 \mathrm{~m}$, we get $1 / \mathrm{Ek} \sim 10000$ at the midlatitudes and 1/Ek $\sim 3000$ in the equatorial regions. Hence, one can expect that a Gaussian submesoscale anticyclone $(\mathrm{Bu}=4)$ remains stable to centrifugal-inertial instability if its intensity does not exceed $|\mathrm{Ro}| \sim 0.52$ at midlatitudes or $|\mathrm{Ro}| \sim 0.65$ in the equatorial regions. The corresponding core vorticity of such stable anticyclones could reach values up to $\zeta(0) \sim-1.7 f$, or $\zeta(0) \sim-2 f$ close to the equator. For weaker diapycnal diffusivity, when $\kappa_{z} \sim \nu \sim 10^{-5} \mathrm{~m}^{2} \mathrm{~s}^{-1}$ a Gaussian anticyclone will still remain stable even if the core vorticity reaches intense values up to $\zeta(0) \sim-1.4 f$ [or $\zeta(0) \sim-1.6 f$ ].

Are such high vorticity values observed for oceanic anticyclones? Unfortunately, observations of submesoscale eddies are sparse since they require high-resolution measurements to be detected. As already mentioned, only HFR measurements or glider transects are able to quantify accurately the velocity profile of small-scale eddies having characteristic radii below $10-15 \mathrm{~km}$. The first quantitative observation of an anticyclonic eddy having negative absolute vorticity for several days was performed in 2002 in the lee of Oahu island in the Hawaiian archipelago (Chavanne et al. 2010). The surface vorticity reaches an extremum of $-1.7 f$ in the eddy core, but the temporal evolution of radial profiles of vorticity was inconsistent with the angular momentum redistribution induced by centrifugalinertial instability (Kloosterziel et al. 2007; Lazar et al. 2013a). More recently, an intense effort of in situ observations has been carried out in the northwestern Mediterranean Sea during four consecutive winters (2010-13). Thanks to autonomous gliders several submesoscale coherent vortices (SCVs) characterized by a small radius (5-8 km) were detected (Bosse et al. 2016). A few of them correspond to intense surface anticyclones with a vortex Rossby number ranging between $|\mathrm{Ro}| \sim 0.27$ and $|\mathrm{Ro}| \sim 0.45$. If we assume a Gaussian velocity profile, the corresponding core vorticity would be between $\zeta(0) \sim-0.9 f$ and $\zeta(0) \sim-1.5 f$. Moreover, several density profiles were available inside and outside these anticyclones allowing for an accurate estimation of the local deformation radius $R_{d}$ and therefore the Burger number. The characteristic values of these few intense surface anticyclones are summarized in Table 1, and a comparison with the present estimated marginal stability curves is plotted in Fig. 14.

To perform a quantitative comparison with the marginal stability limit, we have to estimate the characteristic depth $H$ of these eddies. We have computed $H$ from the standard $e$-folding depth, according to the relation $V(z=-H)=V_{\max } / e$. This yields typical values from $H \sim 100 \mathrm{~m}$ for the surface intensified Oahu anticyclone to $H \sim 300-400 \mathrm{~m}$ for the deeper northwestern Mediterranean eddies where strong mistral winds and surface heat losses deepen the surface mixed layer. Then, we have assumed that the diapycnal diffusivity, induced by the surface winds is on the order of $\kappa_{z} \sim 10^{-4} \mathrm{~m}^{2} \mathrm{~s}^{-1}$. This gives typical Ekman numbers of the order of Ek $\sim 3-$ $5 \times 10^{-4}$ for the thin Hawaiian anticyclone and $\mathrm{Ek} \sim 10^{-5}$ for the thicker Mediterranean SCV. The corresponding marginal stability curves are then plotted in the (Ro, $\sqrt{\mathrm{Bu}}$ ) parameter space (see Fig. 14) and compared to the dynamical values of the few intense anticyclones obtained from in situ measurements. Most of these intense surface anticyclones are in the stable region of the parameter space. Of course this stability analysis should be taken with care, because in situ observations have strong errors bars and the marginal stability curve is quite sensitive to the estimated value of the turbulent viscosity $\kappa_{z} \sim \nu$. Besides, the fact that these intense eddies were observed for several days is a necessary but not sufficient condition for their stability. However, this comparison shows that our stability analysis, unlike the inviscid Rayleigh criterion, provides here a realistic estimation of the marginal stability limit for the 


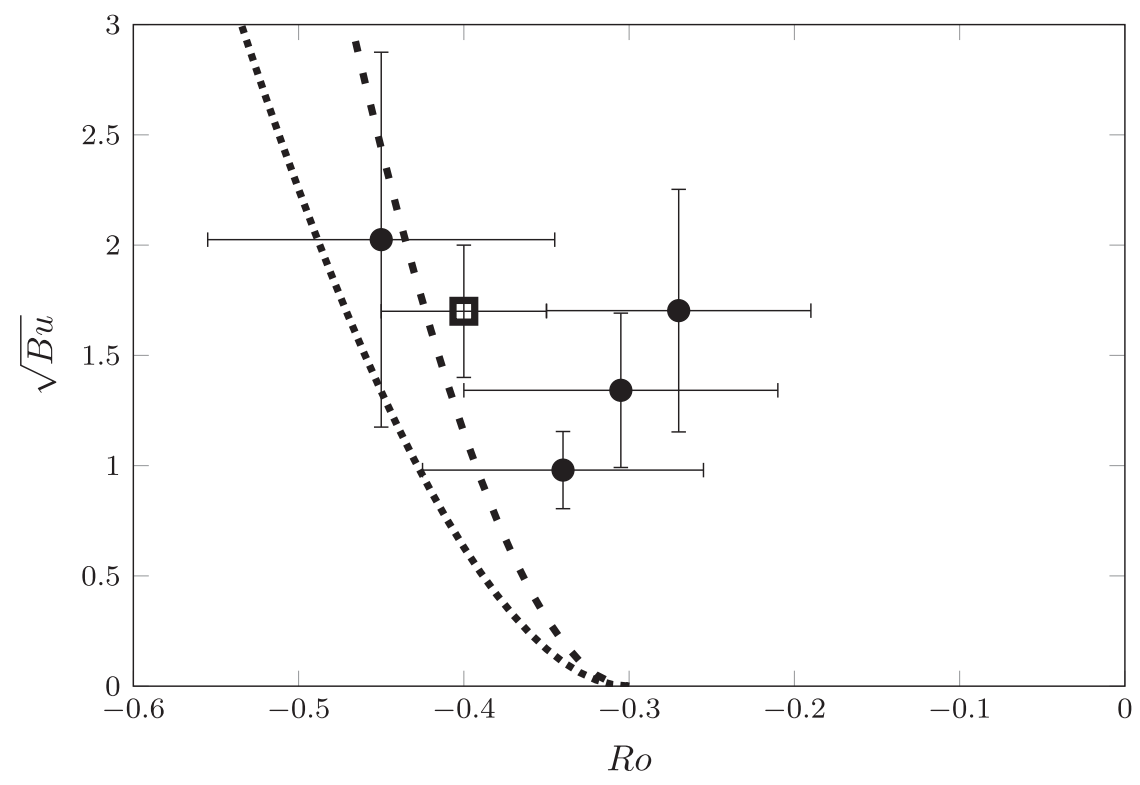

FIG. 14. Location of the intense anticyclone observed in 2002 in the lee of Oahu (square) or the few surface anticyclonic SCV detected in the Mediterranean Sea (circles) plotted in the (Ro, $\sqrt{\mathrm{Bu}}$ ) parameter space. The marginal stability curves (46) for the centrifugal-inertial instability of Gaussian eddies are plotted with a dotted (dashed) line for Ek $=1 / 30000$ $(\mathrm{Ek}=1 / 100000)$.

centrifugal-inertial instability that does not contradict the in situ observations.

\section{APPENDIX A}

\section{Effect of the Aspect Ratio}

Figures A1a and A1b show the effect of the aspect ratio $\delta$ in the case of the GG profile for the fixed parameters $\mathrm{Ro}=-1, \mathrm{Bu}=4$, and $\mathrm{Ek}=1 / 2500$. The growth rate of the azimuthal wavenumbers $m=0,1,2$ and the corresponding vertical wavenumbers $k_{\max }$ is independent of the aspect ratio when $\delta \leq 1$. In contrast, the growth rate decreases when $\delta>1$. Such damping is due to the horizontal viscous dissipation, which scales like $\delta^{2}$ for a fixed Ekman number as seen in (22)-(25). Thus, when $\delta>1$, the horizontal dissipation is enhanced and dominates the vertical dissipation. This effect of the aspect ratio is absent in the asymptotic expression (36) of the growth rate because (30) takes into account only the vertical dissipation and neglects the horizontal dissipation. It could be however possible to take the latter effect into account by adding in (30) a damping term scaling like $\mathrm{Ek} \delta^{2}$. A similar behavior is obtained for $\mathrm{Bu}=1$ and $\mathrm{Ro}=-0.7$ as seen in Figs. A1c and A1d. In conclusion, the aspect ratio has no effect as long as $\delta \leq 1$. The present results for $\delta=0.5$ can be therefore applied to oceanic vortices with $\delta \ll 1$.

\section{APPENDIX B}

\section{Other Types of Instabilities}

In addition to the centrifugal instability investigated in the paper, there exist other types of instability that are subdominant except when the centrifugal instability is stable or marginally unstable. Since these instabilities have been described by Yim et al. (2016) and Mahdinia et al. (2017), we show here only some examples. As shown by Yim et al. (2016), the eigenmodes and frequency ranges corresponding to these other instabilities are very different from those of the centrifugal instability. Figure B1a shows that growth rates of the other instabilities for the G3G profile for $\mathrm{Bu}=4$ near the critical Rossby number of the centrifugal instability. There exist an $m=2$ baroclinic-shear instability (gray dashed line with + symbols) and an $m=1$ GentMcWilliams instability (dashed line with $\times$ symbols). The former instability is called "baroclinic-shear" because its eigenmode resembles the one of the shear instability but its energy source is the potential energy instead of the kinetic energy (Yim et al. 2016). In addition, the two instabilities transform continuously one into the other when the control parameters are varied. The Gent-McWilliams instability occurs for the $m=1$ azimuthal wavenumber only (Gent and McWilliams 1986; Yim and Billant 2015) and bends the vortex. Although 

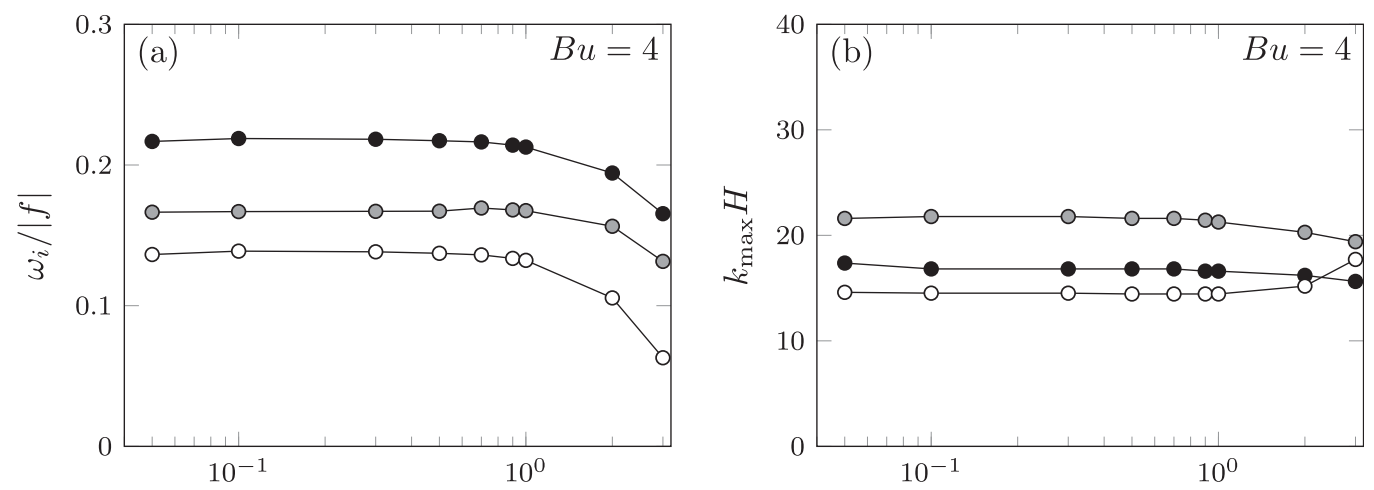

$\delta$
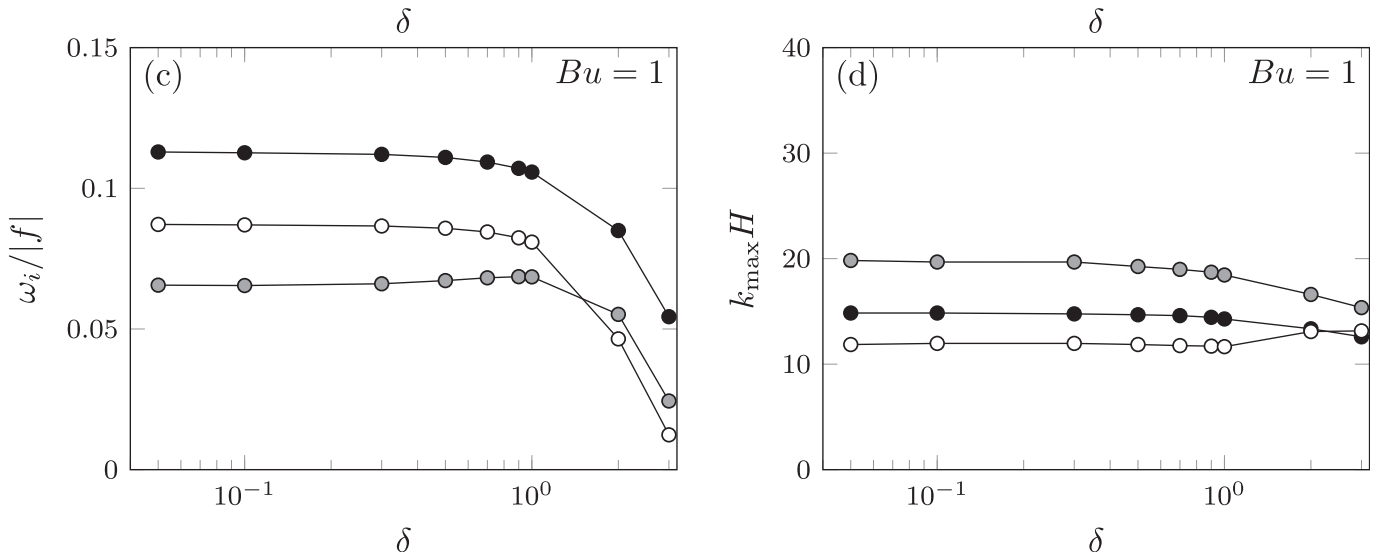

FIG. A1. (left) Maximum growth rate and (right) most amplified vertical wavenumber $k_{\max } H$ as a function of the aspect ratio $\delta$ for $\mathrm{Ek}=1 / 2500$. The different symbols indicate the azimuthal wavenumber: $m=0$ (gray symbols), $m=1$ (black symbols), and $m=2$ (empty symbols) for (a),(b) Ro $=-1, \mathrm{Bu}=4$ and (c),(d) $\mathrm{Ro}=-0.7, \mathrm{Bu}=1$.

it can be continuously connected to the $m=1$ centrifugal instability for some Rossby numbers, for the range of negative Rossby numbers of Fig. B1 it is distinct from the centrifugal instability and thus easily distinguishable (Yim et al. 2016). For Ro $\leq-0.7$, the growth rate of the centrifugal instability (black solid lines) rises quickly and becomes dominant. A similar evolution is shown in Fig. $\mathrm{B} 1 \mathrm{~b}$ for $\mathrm{Bu}=25$. The baroclinic-shear instability is dominant only when Ro is near or above the critical Rossby number of the centrifugal instability. Note that in Fig. B1a the growth rate is scaled by $\Omega_{0}$ instead of $|f|$. Otherwise, the scaled growth rates would vanish for
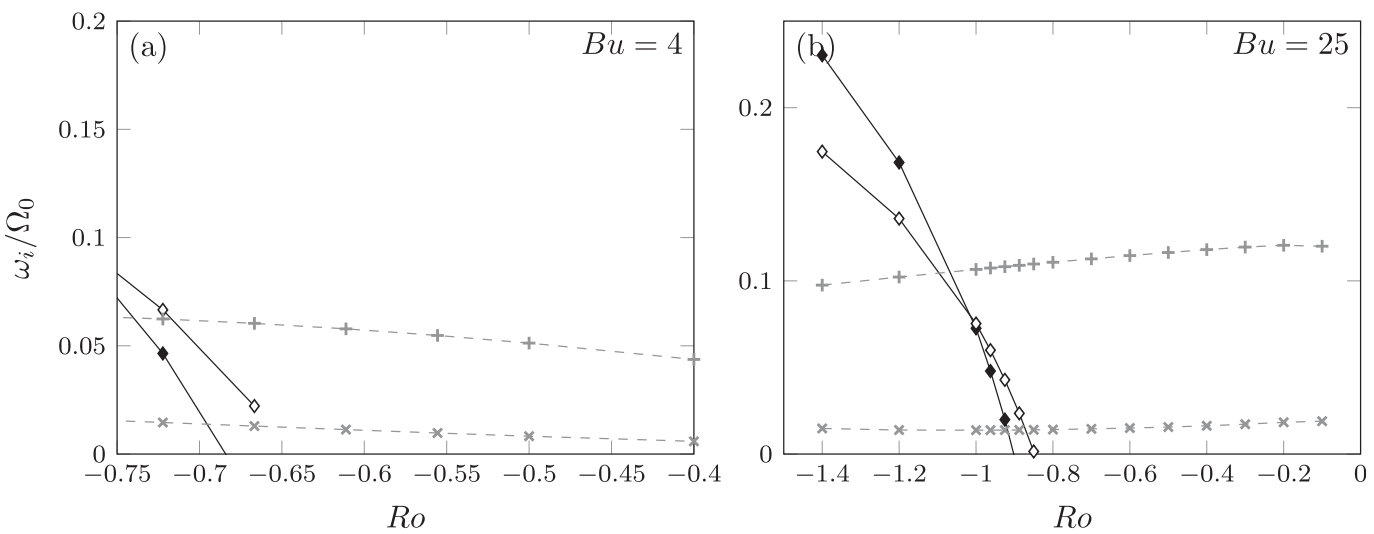

FIG. B1. Growth rate of different instabilities for the G3G eddy for $\delta=0.5, \mathrm{Ek}=1 / 2500$ for (a) $\mathrm{Bu}=4$ and (b) $\mathrm{Bu}=25$. The black solid lines indicate the growth rate of the centrifugal instability and the different symbols represent azimuthal wavenumbers: $m=1$ (black symbols) and $m=2$ (empty symbols). The dashed lines with symbols show the growth rate of the different instabilities: $m=1$ Gent-McWilliams instability $(\times)$ and $m=2$ baroclinic-shear instability $(+)$. 
small $\mid$ Ro $\mid$ since $|f|$ increases. This allows us to show that the growth rate of the baroclinic-shear and GentMcWilliams instabilities are almost independent of Ro in the range investigated.

\section{APPENDIX C}

\section{Expressions of the Coefficients of the Asymptotic Analysis of Section 4d}

Here, we give the detailed expressions of the coefficients in (40)-(42):

$$
\begin{aligned}
& a_{1}=\frac{m \alpha \sqrt{m^{2}-2 \alpha-4}-m^{2}(\alpha+4)+2 \alpha^{2}+12 \alpha+16}{2(\alpha+2)\left(-m^{2}+2 \alpha+4\right)}, \\
& c_{1}=\operatorname{Im}\left(-a_{1} m+2 i c_{2}\right),
\end{aligned}
$$

where $\operatorname{Im}(\cdot)$ indicates the imaginary part and

$$
c_{2}=\sqrt{\left(1-a_{1}\right)\left[\frac{a_{1}}{2}(\alpha+2)-1\right]} .
$$

The coefficient $c_{3}$ is given by

$$
c_{3}=\operatorname{Im}\left(\frac{i \sqrt{\frac{a_{1}}{2} \alpha\left\{-a_{1}\left[\left(m^{2}-6\right) \alpha-4 \alpha^{2}+4\right]-(\alpha-1)\left(2 i m c_{2}+\alpha+4\right)\right\}}}{2^{3 / 2} c_{2}\left(a_{1} \alpha\right)^{1 / \alpha}}\right) .
$$

Finally, $c_{4}$ reads

$$
c_{4}=\left(\frac{c_{1}}{3 e^{-1 / \alpha}}\right)^{3 / 2} \frac{2}{c_{3}} .
$$

\section{REFERENCES}

Ash, R. L., and M. R. Khorrami, 1995: Vortex stability. Fluid Vortices, S. I. Green, Ed., Fluid Mechanics and Its Applications, Vol. 30, 317-372, https://doi.org/10.1007/978-94-011-0249-0_8.

Balay, S., and Coauthors, 2014: PETSc users manual. Tech. Rep. ANL-95/11, Revision 3.5, Argonne National Laboratory, 271 pp., https:/www.mcs.anl.gov/petsc/documentation/index.html.

Batchelor, G. K., and A. E. Gill, 1962: Analysis of the stability of axisymmetric jets. J. Fluid Mech., 14, 529-551, https://doi.org/ 10.1017/S0022112062001421.

Benilov, E., 2003: Instability of quasi-geostrophic vortices in a twolayer ocean with a thin upper layer. J. Fluid Mech., 475, 303331, https://doi.org/10.1017/S0022112002002823.

Billant, P., and F. Gallaire, 2005: Generalized Rayleigh criterion for non-axisymmetric centrifugal instabilities. J. Fluid Mech., 542, 365-379, https://doi.org/10.1017/S0022112005006464.

_- A. Colette, and J.-M. Chomaz, 2004: Instabilities of a vortex pair in a stratified and rotating fluid. Proc. 21st Int. Congress of Theoretical and Applied Mechanics, Warsaw, Poland, IUTAM, 16-20.

Bosse, A., and Coauthors, 2016: Scales and dynamics of submesoscale coherent vortices formed by deep convection in the northwestern Mediterranean Sea. J. Geophys. Res. Oceans, 121, 7716-7742, https://doi.org/10.1002/2016JC012144.

Capet, X., J. C. McWilliams, M. J. Molemaker, and A. F. Shchepetkin, 2008: Mesoscale to submesoscale transition in the California Current System. Part I: Flow structure, eddy flux, and observational tests. J. Phys. Oceanogr., 38, 29-43, https://doi.org/10.1175/2007JPO3671.1.

Carnevale, G. F., and R. C. Kloosterziel, 1994: Emergence and evolution of triangular vortices. J. Fluid Mech., 259, 305-331, https://doi.org/10.1017/S0022112094000157.
Carton, X. J., and J. C. McWilliams, 1989: Barotropic and baroclinic instabilities of axisymmetric vortices in a quasigeostrophic model. Mesoscale/Synoptic Coherent Structures in Geophysical Turbulence, J. Nihoul and B. Jamart, Eds., Elsevier Oceanography Series, Vol. 50, Elsevier, 225-244.

Chavanne, C., P. Flament, and K.-W. Gurgel, 2010: Interactions between a submesoscale anticyclonic vortex and a front. J. Phys. Oceanogr., 40, 1802-1818, https://doi.org/10.1175/ 2010JPO4055.1.

Eliassen, A., and E. J. Kleinschmidt, 1957: Dynamic meteorology. Geophysik II, J. Bartels, Ed., Handbuch der Physik, Vol. 10/48, Springer, 1-154, https://doi.org/10.1007/978-3-642-45881-1_1.

Elman, H., D. Silvester, and A. Wathen, 2005: Finite Elements and Fast Iterative Solvers: With Applications in Incompressible Fluid Dynamics. Oxford University Press, 416 pp.

Facchini, G., and M. Le Bars, 2016: On the lifetime of a pancake anticyclone in a rotating stratified flow. J. Fluid Mech., 804, 688-711, https://doi.org/10.1017/jfm.2016.549.

Flierl, G. R., 1988: On the instability of geostrophic vortices. J. Fluid Mech., 197, 349-388, https://doi.org/10.1017/ S0022112088003283.

Garabato, A. C. N., K. L. Polzin, B. A. King, K. J. Heywood, and M. Visbeck, 2004: Widespread intense turbulent mixing in the Southern Ocean. Science, 303, 210-213, https://doi.org/ 10.1126/science.1090929.

Garnaud, X., 2012: Modes, transient dynamics and forced response of circular jets. Ph.D. thesis, LadHyX, Ecole Polytechnique X, $144 \mathrm{pp}$.

— , L. Lesshafft, P. J. Schmid, and P. Huerre, 2013: Modal and transient dynamics of jet flows. Phys. Fluids, 25, 044103, https://doi.org/10.1063/1.4801751.

Gent, P. R., and J. C. McWilliams, 1986: The instability of barotropic circular vortices. Geophys. Astrophys. Fluid Dyn., 35 , 209-233, https://doi.org/10.1080/03091928608245893.

Gula, J., M. J. Molemaker, and J. C. McWilliams, 2015: Gulf Stream dynamics along the southeastern U.S. seaboard. J. Phys. Oceanogr., 45, 690-715, https://doi.org/10.1175/JPO-D-14-0154.1.

Hasegawa, D., H. Yamazaki, R. G. Lueck, and L. Seuront, 2004 How islands stir and fertilize the upper ocean. Geophys. Res. Lett., 31, L16303, https://doi.org/10.1029/2004GL020143. 
Hecht, F., 2012: New development in freefem ++. J. Numer. Math., 20, 251-265, https://doi.org/10.1515/jnum-2012-0013.

Helfrich, K. R., and U. Send, 1988: Finite-amplitude evolution of two-layer geostrophic vortices. J. Fluid Mech., 197, 331-348, https://doi.org/10.1017/S0022112088003271.

Hernandez, V., J. E. Roman, and V. Vidal, 2005: SLEPc: A scalable and flexible toolkit for the solution of eigenvalue problems. ACM Trans. Math. Software, 31, 351-362, https://doi.org/ 10.1145/1089014.1089019.

Hua, B. L., C. Ménesguen, S. Le Gentil, R. Schopp, B. Marsset, and H. Aiki, 2013: Layering and turbulence surrounding an anticyclonic oceanic vortex: In situ observations and quasigeostrophic numerical simulations. J. Fluid Mech., 731, 418442, https://doi.org/10.1017/jfm.2013.369.

Ikeda, M., 1981: Instability and splitting of mesoscale rings using a twolayer quasi-geostrophic model on an $f$-plane. J. Phys. Oceanogr., 11, 987-998, https://doi.org/10.1175/1520-0485(1981)011<0987: IASOMR $>2.0 . \mathrm{CO} ; 2$

Ioannou, A., A. Stegner, B. Le Vu, I. Taupier-Letage, and S. Speich, 2017: Dynamical evolution of intense Ierapetra eddies on a 22 year long period. J. Geophys. Res. Oceans, $\mathbf{1 2 2}$, 9276-9298, https://doi.org/10.1002/2017JC013158.

Johannessen, J., G. Digranes, H. Espedal, O. Johannessen, P. Samuel, D. Browne, and P. Vachon, 1994: SAR ocean feature catalogue. European Space Agency Spec. Publ. 1174, 106 pp.

Klein, P., B. L. Hua, G. Lapeyre, X. Capet, S. Le Gentil, and H. Sasaki, 2008: Upper ocean turbulence from high-resolution 3D simulations. J. Phys. Oceanogr., 38, 1748-1763, https:// doi.org/10.1175/2007JPO3773.1.

Kloosterziel, R. C., and G. J. F. van Heijst, 1991: An experimental study of unstable barotropic vortices in a rotating fluid. J. Fluid Mech., 223, 1-24, https://doi.org/10.1017/S0022112091001301.

_, G. Carnevale, and P. Orlandi, 2007: Inertial instability in rotating and stratified fluids: Barotropic vortices. J. Fluid Mech., 583, 379412, https://doi.org/10.1017/S0022112007006325.

Kunze, E., and J. Toole, 1997: Tidally driven vorticity, diurnal shear, and turbulence atop Fieberling Seamount. J. Phys. Oceanogr., 27, 2663-2693, https://doi.org/10.1175/1520-0485 (1997)027<2663:TDVDSA $>2.0$. CO;2.

Lahaye, N., and V. Zeitlin, 2015: Centrifugal, barotropic and baroclinic instabilities of isolated ageostrophic anticyclones in the two-layer rotating shallow water model and their nonlinear saturation. J. Fluid Mech., 762, 5-34, https://doi.org/10.1017/jfm.2014.631.

Lazar, A., A. Stegner, and E. Heifetz, 2013a: Inertial instability of intense stratified anticyclones. Part 1 . Generalized stability criterion. J. Fluid Mech., 732, 457-484, https://doi.org/10.1017/jfm.2013.412.

$\longrightarrow,-$, R. Caldeira, C. Dong, H. Didelle, and S. Viboud, 2013b: Inertial instability of intense stratified anticyclones. Part 2. Laboratory experiments. J. Fluid Mech., 732, 485-509, https:// doi.org/10.1017/jfm.2013.413.

Leblanc, S., and C. Cambon, 1997: On the three-dimensional instabilities of plane flows subjected to Coriolis force. Phys. Fluids, 9, 1307-1316, https://doi.org/10.1063/1.869273.

Ledwell, J., A. Watson, and C. S. Law, 1998: Mixing of a tracer in the pycnocline. J. Geophys. Res., 103, 21 499-21 529, https:// doi.org/10.1029/98JC01738.

Mahdinia, M., P. Hassanzadeh, P. S. Marcus, and C.-H. Jiang, 2017: Stability of three-dimensional Gaussian vortices in an unbounded, rotating, vertically stratified, Boussinesq flow: Linear analysis. J. Fluid Mech., 824, 97-134, https://doi.org/10.1017/jfm.2017.303.

Munk, W., L. Armi, K. Fischer, and F. Zachariasen, 2000: Spirals on the sea. Proc. Roy. Soc. London, 456A, 1217-1280, https:// doi.org/10.1098/rspa.2000.0560.
Mutabazi, I., C. Normand, and J. E. Wesfreid, 1992: Gap size effects on centrifugally and rotationally driven instabilities. Phys. Fluids, 4A, 1199-1205, https://doi.org/10.1063/1.858238.

Negretti, M. E., and P. Billant, 2013: Stability of a Gaussian pancake vortex in a stratified fluid. J. Fluid Mech., 718, 457-480, https://doi.org/10.1017/jfm.2012.624.

Nguyen, H. Y., B. L. Hua, R. Schopp, and X. Carton, 2012: Slow quasigeostrophic unstable modes of a lens vortex in a continuously stratified flow. Geophys. Astrophys. Fluid Dyn., 106, 305-319, https://doi.org/10.1080/03091929.2011.620568.

Orlandi, P., and G. F. Carnevale, 1999: Evolution of isolated vortices in a rotating fluid of finite depth. J. Fluid Mech., 381, 239-269, https://doi.org/10.1017/S0022112098003693.

Paduan, J. D., and L. Washburn, 2013: High-frequency radar observations of ocean surface currents. Аnnu. Rev. Mar. Sci., 5, 115-136, https://doi.org/10.1146/annurev-marine-121211-172315.

Plougonven, R., and V. Zeitlin, 2009: Nonlinear development of inertial instability in a barotropic shear. Phys. Fluids, 21, 106601, https://doi.org/10.1063/1.3242283.

Rayleigh, L., 1917: On the dynamics of revolving fluids. Proc. Roy. Soc. London, 93A, 148-154, https://doi.org/10.1098/rspa.1917.0010.

Reinaud, J. N., 2017: Piecewise uniform potential vorticity pancake shielded vortices. Geophys. Astrophys. Fluid Dyn., 111, 32-64, https://doi.org/10.1080/03091929.2016.1275609.

Schaeffer, A., A. Gramoulle, M. Roughan, and A. Mantovanelli, 2017: Characterizing frontal eddies along the East Australian Current from HF radar observations. J. Geophys. Res. Oceans, 122, 3964-3980, https://doi.org/10.1002/2016JC012171.

Sipp, D., and L. Jacquin, 2000: Three-dimensional centrifugal-type instabilities of two-dimensional flows in rotating systems. Phys. Fluids, 12, 1740-1748, https://doi.org/10.1063/1.870424.

Smyth, W. D., and J. C. McWilliams, 1998: Instability of an axisymmetric vortex in a stably stratified, rotating environment. Theor. Comput. Fluid Dyn., 11, 305-322, https://doi.org/ 10.1007/s001620050095.

Solberg, H., 1936: Le mouvement d'inertie de l'atmosphère stable et son rôle dans la théorie des cyclones. Proc. Union Geodesique et Geophysique Internationale Vieme Assemblee, Edinburgh, United Kingdom, UGGI, 66-82.

Stegner, A., and D. Dritschel, 2000: A numerical investigation of the stability of isolated shallow water vortices. J. Phys. Oceanogr., 30, 2562-2573, https://doi.org/10.1175/1520-0485(2000) $030<2562$ :ANIOTS $>2.0$.CO;2.

Sutyrin, G., and T. Radko, 2017: The fate of pancake vortices. Phys. Fluids, 29, 031701, https://doi.org/10.1063/1.4977975.

Thompson, A., S. Gille, J. A. MacKinnon, and J. Sprintall, 2007: Spatial and temporal patterns of small-scale mixing in Drake Passage. J. Phys. Oceanogr., 37, 572-592, https://doi.org/ 10.1175/JPO3021.1.

Yim, E., 2015: Stability of columnar and pancake vortices in stratified-rotating fluids. Ph.D. thesis, LadHyX, Ecole Polytechnique $\mathrm{X}, 176 \mathrm{pp}$.

—, and P. Billant, 2015: On the mechanism of the GentMcWilliams instability of a columnar vortex in stratified rotating fluids. J. Fluid Mech., 780, 5-44, https://doi.org/ 10.1017/jfm.2015.426.

, and - 2016: Analogies and differences between the stability of an isolated pancake vortex and a columnar vortex in stratified fluid. J. Fluid Mech., 796, 732-766, https://doi.org/ 10.1017/jfm.2016.248.

, - — and C. Ménesguen, 2016: Stability of an isolated pancake vortex in continuously stratified-rotating fluids. J. Fluid Mech., 801, 508-553, https://doi.org/10.1017/jfm.2016.402. 